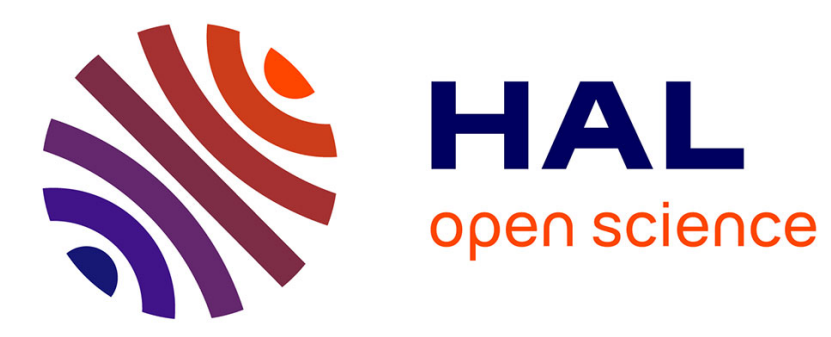

\title{
Résultats historiques des fouilles d'Entremont (1946-1967)
}

\author{
Fernand Benoit
}

\section{To cite this version:}

Fernand Benoit. Résultats historiques des fouilles d'Entremont (1946-1967). Gallia - Fouilles et monuments archéologiques en France métropolitaine, 1968, 26 (1), pp.1-31. 10.3406/galia.1968.2490 . hal-01934419

\section{HAL Id: hal-01934419 https://hal.science/hal-01934419}

Submitted on 3 Mar 2020

HAL is a multi-disciplinary open access archive for the deposit and dissemination of scientific research documents, whether they are published or not. The documents may come from teaching and research institutions in France or abroad, or from public or private research centers.
L'archive ouverte pluridisciplinaire HAL, est destinée au dépôt et à la diffusion de documents scientifiques de niveau recherche, publiés ou non, émanant des établissements d'enseignement et de recherche français ou étrangers, des laboratoires publics ou privés.

\section{(이) $\$$}

Distributed under a Creative Commons Attribution - NonCommercial - NoDerivatives| 4.0 


\title{
RÉSULTATS HISTORIQUES DES FOUILLES D'ENTREMONT $(1946-1967)$
}

\author{
par Fernand BENOIT
}

La mise au jour, commencée en 1946 avec la collaboration de M. R. Ambard ${ }^{1}$, de l'oppidum d'Entremont, dont le sommet est toujours occupé par l'Autorité militaire, a dégagé, à la date de 1967, une partie des deux enceintes, l'une limitant la ville au nord dans sa plus grande extension (Entremont III), l'autre délimitant un quartier intérieur (Entremont II), deux groupes d'habitations correspondant à chacun de ces quartiers (plan, fig. 1) et les éléments d'architecture et de sculpture de sanctuaires : le plus ancien (que nous désignons sous le nom d'Entremont I), non encore localisé, comprenait des piliers et des stèles aniconiques, qui ont été retrouvés en remploi, dans la construction du rempart d'Entremont II ; le "sanctuaire aux crânes" avait été aménagé entre deux tours de ce rempart et la statuaire ${ }^{2}$ était groupée le long de la "voie sacrée " qui se prolonge dans le terrain militaire, à l'ouest de ce sanctuaire.

La ville, polis, ainsi que l'appelle Diodore de Sicile, Inlermonles au Moyen Age, à $3 \mathrm{~km}$ au nord d'Aix, occupait un plateau en forme d'éperon triangulaire, en légère pente vers le nord, défendu par une enceinte sur cette face et protégé par l'escarpement rocheux sur les autres faces. Elle apparaît divisée en deux aires d'inégale superficie : l'une au sud, rectangulaire, la "ville haute" (Entremont II), couvrait la partie la plus élevée du plateau (altitude 368-367 m), défendue sur les faces sud-est et sud-ouest par la falaise et sur ses deux autres faces par un rempart dont le front nord n'a pu être que partiellement dégagé, étant en partic dans le terrain militaire (parcelle 3407), et dont le front est, reconnaissable à un alignement de grands blocs, assis sur le rebord du plateau, domine de quelque 2 à 3 mètres la "ville basse" (Entremont III). Celle-ci, à une altitude moyenne de 364-358 m,

(1) Voir les chroniques archéologiques donnant le compte rendu des fouilles dans Gallia, v, 1947, pp. 81-97 : VIII, 1950, pp. 117-119; XII, 1954, pp. 285-294; XIV, 1956, pp. 218-222; XVI, 1958, pp. 412-415; XVIII, 1960, pp. 291-294; XX, 1962, pp. 689-692; XXII, 1964, pp. 573-575.

(2) F. Bevort, L'Arl primilif méditerranéen, $2^{\mathrm{e}}$ édit., 1955, 73 p., $64 \mathrm{pl}$; La statuaire d'Entremont, dans VIII $\mathrm{e}$ Congrès intern. archéolngie classique, Paris, 1963 (1965), p. 655; Espérandec-LaNtier, Recueil des bas-reliefs..., 15 (1966), 8652-8682. 


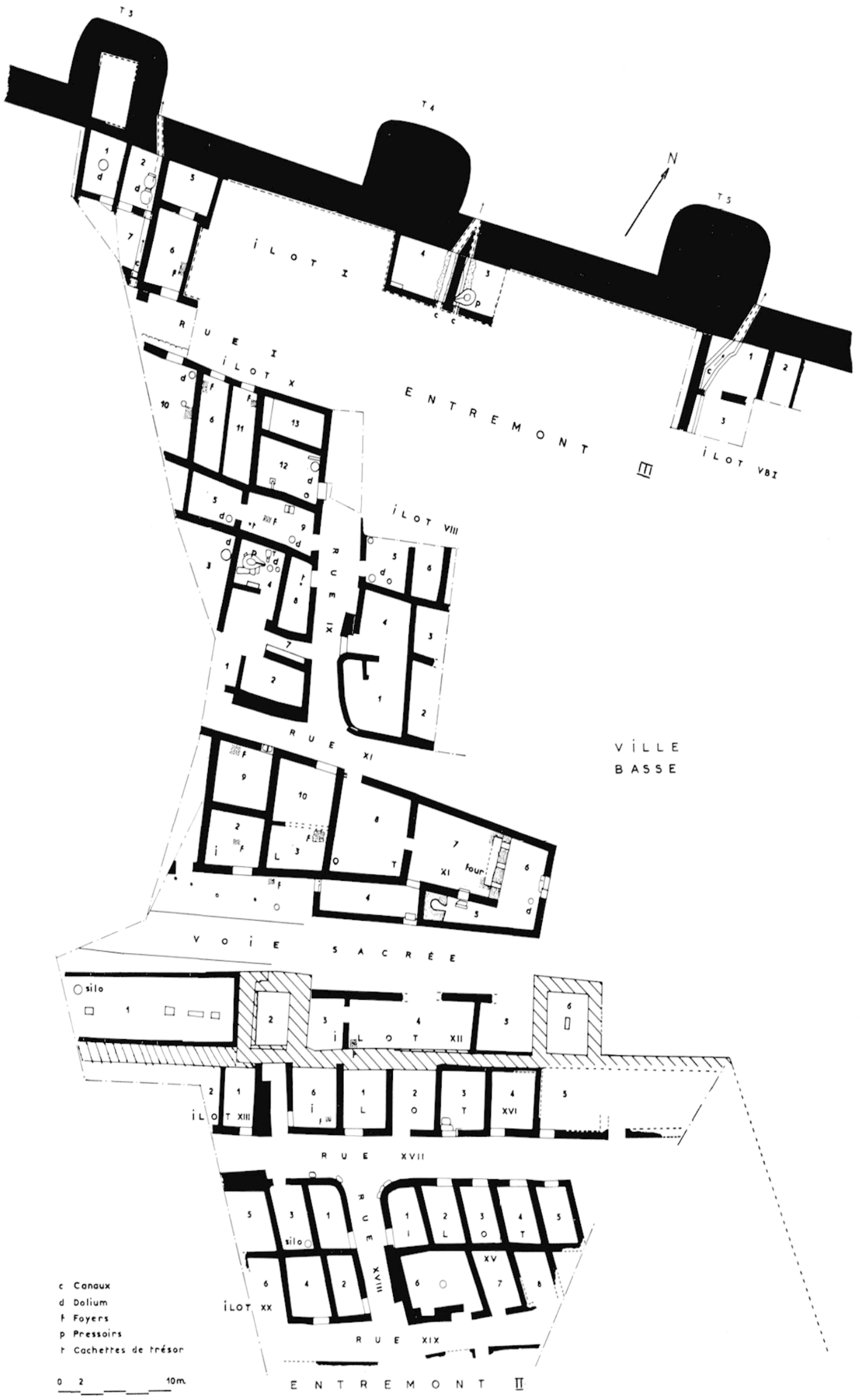

1 Plan partiel des deux quartiers d'Entremont II et III avec leur enceinte, limitrophes du terrain militaire. (Releve R. Ambard, P. Pironin et M. Borély). 
occupait la pointe du triangle et s'étendait au nord jusqu'à l'enceinte qui barrait l'ensemble du plateau. Cette division bipartite de la ville pose un problème qui ne sera résolu que lorsque la fouille de la ville haute pourra ètre étendue rlans le secteur encore occupé par l'Autorité militaire.

La juxtaposition d'un romptoir massaliète et d'une ville indigène ne pouvant être retenue, et en l'absence de tout critère chronologique fourni par la céramique, qui est identique dans les deux quartiers, ou par la terhnique de la construction des cases, qui ont été restaurées à plusieurs reprises, on ne peut qu'émettre une hypothèse de travail : le développement de la ville s'inserit dans un court intervalle de temps, entre le début du

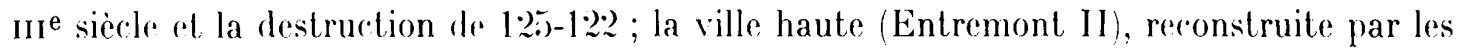
Salyens, aurait eu une destination fonctionnelle et sociale; elle aurait été le quartier de résidener des rois et princes salyens, dynaslai et principes que nous font connaître les récits de la prise de la ville, avec casernements et greniers (îlots XV et XVI). dont la plupart des cases sont dépourvues de foyers, ce que rendrait vraisemblable le grand nombre de dolia retrouvés brisés dans la rue après le pillage; ou bien ce quartier serait l'ancien oppidum ligure, englobé à une époque postérieure dans une grande ville (Entremont III). En ce cas, cette extension ne serait-elle pas due à la conquête de la basse Provence occidentale par les Celtes, qui navaient franchi la Durance que vers le milieu du in e siècle ? Se mêlant étroitement aux tribus ligures, d'où le nom de "Celto-Ligures" que leur donne Strabon, ils avaient fait d'Entremont la capitale d'une puissante confédération militaire, les Salyens ou Salluvii, qui s'étendait du Rhône jusqu'aux montagnes des Vlaures, que tenait encore la tribu ligure des Oxybiens, maîtres de la vallée de l'Argens.

La superficie de la ville d'lintremont (3 ha $1 / 2$ ) contraste en effet avec l'exiguïté des forteresses ligures, qui constituaient un refuge en cas de danger et un grenier pour les récoltes. Mais la ville n'englobait pas la totalité des habitations. Sur le versant ouest, étagées sur la pente abrupte de la falaise, ont été mises au jour des cases taillées dans le rocher, avec foyer d'argile, selon le mode de l'habitat rupestre signalé à Cavaillon, à Glanum, à Montlaurès. Si l'on tient compte du nombre élevé des habitants (900) au dire de Diodore de Sicile auraient reçu leur gräce du consul), on doit supposer que la ville n'abritait pas toute la tribu, dont une partie étail sans doute semi-nomade et habitait la rampagne.

Elle ne fut pas réoccupée à l'époque romaine et retourna à l'état agreste ; aucun tesson d'Arezzo ou de poterie sigillée, aucune monnaie impériale, que l'on trouve par contre en abondance dans les sites voisins. La mise en culture des terres et surtout l'épierrement du plateau fait au début du siècle (1903), pour niveler la route de Puyricard, avaient fait disparaître toute trace du rempart et de la tour où avait été conclue en 123:3 la paix entre les comtes de Provence et de T'oulouse, sur l'intervention du légat de Frédéric II, roi des Romains ; cette tour encore signalée au XIv siècle, comme tour de guet, au sommet du plateau (Entremont II).

3, On notera quelques debris de legulae et d'imbrices dans la case XVI 4, provenant d'une occupation temporaire a l'époque romaine (iallia, XIV, 1956, p. 2:2: : 


\section{La pRISE de la ville et la soumission des Salyexs}

La soumission des tribus celto-ligures du littoral et de la basse vallée du Rhône, les Ligures, les Voconces, les Salyens, qui avaient l'appui des Allobroges cantonnés dans le Dauphiné, mais aussi des Arvernes, nécessita plusieurs campagnes de 125 à 121. Marseille, inquiète de la menace que faisait peser sur la vallée du Rhône l'impérialisme des Arvernes alliés des Salyens ${ }^{4}$, avait invoqué le concours de Rome, comme elle l'avait fait trente ans aupararant contre les Ligures du littoral de l'Estérel; sans doute, est-ce à cette époque qu'elle édifia une nouvelle enceinte, qui englobait le faubourg (pedeon) mentionné par une scolie de Lucain ${ }^{5}$ et qui sera relevée, après le siège de César, grâce aux libéralités du médecin marseillais, Crinas, établi à Rome à l'époque de Claude et de Néron.

Une première expédition, descendue par les cols des Alpes, fut conduite par l'un des consuls de 125), .I. Fulvius Flaccus, "contre les Ligures, les Voconces et les Salyens", ainsi que l'atteste la célébration de son triomphe à Rome en 123. Ses troupes furent sans doute maintenues dans le pays pendant son proconsulat et coopérèrent en ce cas en 124 avec le nouveau consul, C. Sextius Calvinus, qui amenait de nouvelles légions. Celui-ci resta sur place également deux ans, puisqu'il célébra son triomphe en 122 "contre les Ligures, les Voconces et les Salyens ", l'année même de la fondation d'Aix, Aquae Sexliae Salluviorum ('arte, fig. 2). Si l'on en croit Diodore de Sicile ${ }^{6}$, c'est au cours de son année consulaire, en 124, que fut prise la ville des Salyens : le consul avait libéré Craton, un indigène au nom grec, et ses compagnons au nombre de 900 (?), amis de Marseille et par conséquent collaborateurs de Rome, le reste de la population étant déportée ou vendue à l'encan.

Mais la pacification des tribus ligures n'ètait qu'apparente: Sextius, après la fondation d'Aix, selon Strabon ${ }^{7}$ a son retour, ou, ce qui paraît plus vraisemblable, au cours de sa marche de Cisalpine vers le pays salyen, avait dù ouvrir de nouvelles hostilités contre les Ligures cantonnés sur le littoral, dont le port d'Aegilna (Fréjus ?) avait cependant été détruit en 154 par le consul (Opimius, "afin d'expulser, écrit Strabon, les barbares de tout le littoral qui conduit de .Iarseille en Italie ». Les Salyens avaient trouvé appui auprès des Allobroges, chez qui s'étaient réfugiés le roi Teutomalius et les princes d'Entremont, échappés au vainqueur. l'ne double campagne fut dirigée par les consuls Cinaeus Iomitius Ahenobarbus en 122) contre les Allobroges, et Q. Fabius Maximus en 121 contre les Arvernes, qui, sous la concluite de leur roi Bituit, avaient franchi le Rhône vers Pont-Saint-lssprit8. Ils furent surcessivement anéantis, avec des pertes considérables, au cours de deux combats sur la rive gauche du Rhône, dans la plaine de la Sorgue, près de l'oppidum de Vindalium ${ }^{9}$

4. STRABOs, $11,2,3$

5) F. Broir, Topographie anlique de Marseille, dans Gallia, XXIV, 1966, p. 19.

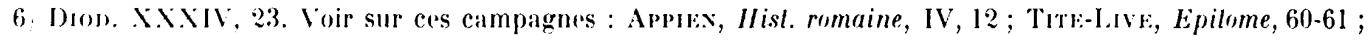

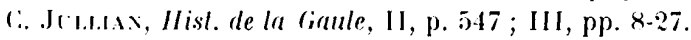

7 ; 1 ; 1,5 et 9 ; et 6,3 .

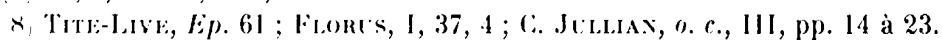

9) Pent-être le Mourre de sive Jeısax, o.c. p. 16, n. 2), où a été localisé le site romain autour de l'oppidum jusqu'à Violiene. 


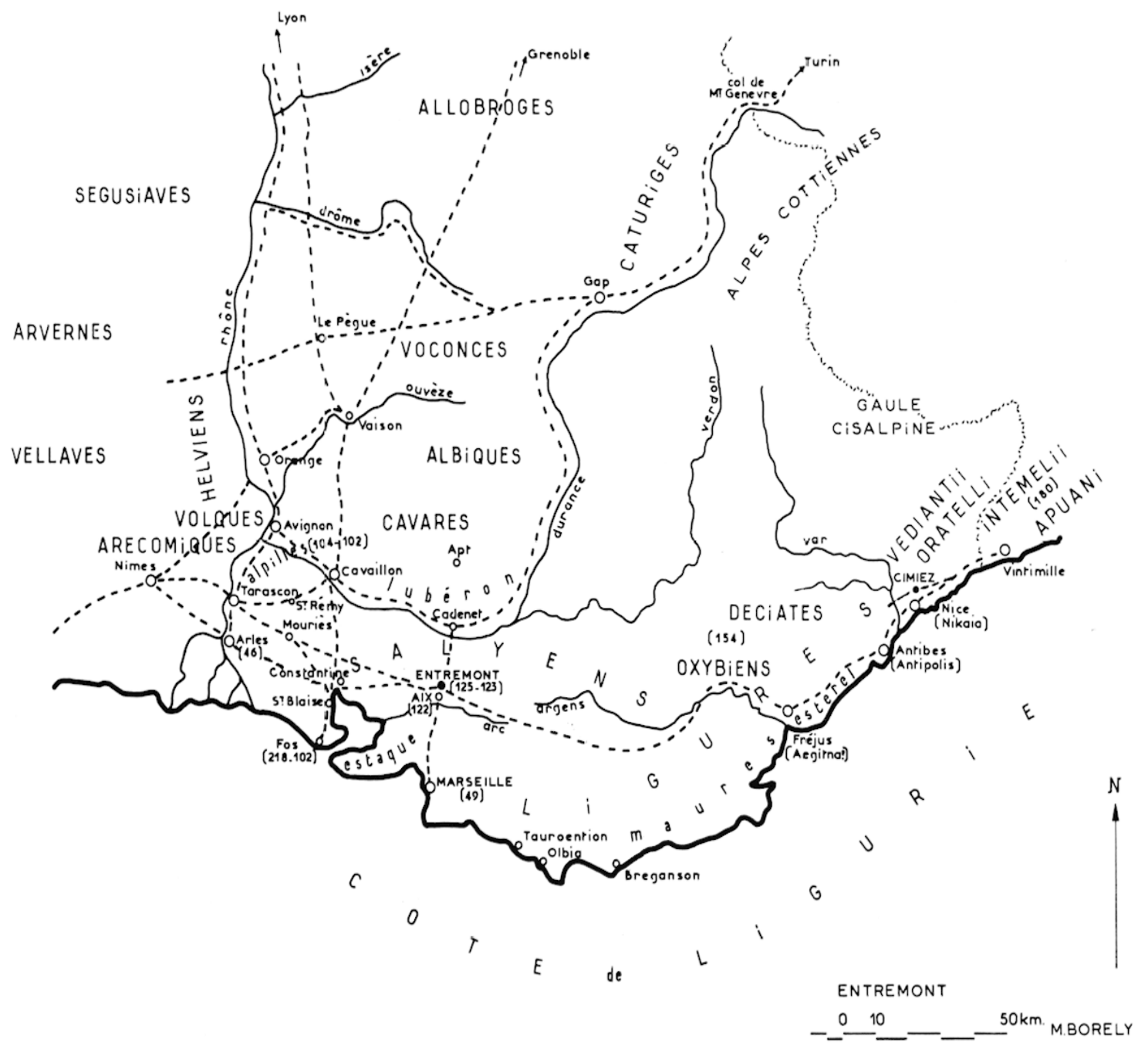

2 Les étapes de la progression romaine : date de soumission des tribus ligures et celto-ligures avec leur situation, et indication des pistes gauloises. (Dessin M. Borély).

et entre Bollène et Orange, peut-être dans la plaine de l'Eygues (Isora, Icarus, Skara) ${ }^{10}$. Les généraux vainqueurs élevèrent un trophée et deux temples à Mars et à Hercule, sur le lieu de la victoire.

La soumission des Salyens paraît avoir été définitive, le pays entre Aix et Arles étant

(10) C. Jullian. o. c., III, p. 17, n. 4. Le nom de cet afluent du Rhỏne qui, avec l'Ouvèze et la Vleyne, formait une ile fertile, avait déjà provoqué une confusion avec l'Isère (Isara), dans le récit de la marche d'Hannibal vers les Alpes en 218 F. Besolt, Les itinéraires commerciaux en Provence dans l'antiquité, dans Assoc. (i. Budé, VII Congrès, Aix-en-Provence, 1963, p. 381). La Iocalisation du camp de Marius au confluent du Rhòne et de l'Isire d'après un texte de Paul Orose, proposée par le Dr A. Doxisantex (Rev. Etudes anciennes, 1954, p. 281) est insoutenable du point de vue stratégique (F. BKolt, dans Provence hislorique, 7, 1957, p. 234) Le trésor de quelque 200 statères des Arvernes découvert à Saint-Uze près de Saint-Vallier, en territoire allobroge, doit-il être daté de cette. bataille, ainsi que le supposait A. Br.ANchet (Rev. numismatique, 1938, p. 125) ? J.-B. Colbert DE: BEACinte, se

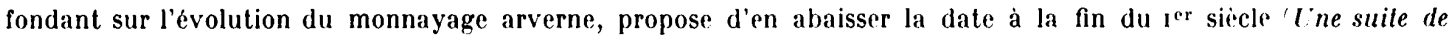
slatires d'or altribues aux Arvernes, dans Rev. Belge de numismatique, 104, 1958, p. 72; Cimlauf und C:hronolngie der gallo-kellischen Münzen, dans Jahrbuch für Numismalik und Geldgeschichle, 1966, p. 47). 
intégré au domaine massaliète. ("est dans leur territoire que Marius établira son camp (101-102), sur la rive gauche de la Durance, sur la croupe des Alpilles, sans doute entre Ernaginum et Glanum ${ }^{11}$, pour attendre l'invasion des Ambrons et des Teutons, qu'il arrêtera dans la plaine d'Aix, sur la voie d'Italie. Par contre, la soumission des Allobroges, la tribu la plus indépendante et la plus puissante de la rive gauche du Rhône, qui tentera de se révolter en 62 et à laquelle Vercingétorix songera à offrir le principat de la nouvelle province romaine ${ }^{12}$, ne sera accomplie qu'après la campagne de César et la défaite des Arvernes, dont l'impérialisme visait au protectorat des tribus de la future Narbonnaise.

Il apparait donc que, si la basse vallée du Rhône fut investie par les légions pendant cinq ans, de 125 à 121, la capitale des Salyens dut aflronter la double campagne des consuls de 125 et de 124. Sans doute subit-elle deux sièges successifs, comme le révèle la stratigraphie du quartier nord. La découverte, en effet, de boulets de lithoboles à deux niveaux différents, séparés par une couche hâtive de remblai (case XII. $4^{13}$, rue IX ct voie sacrée). révèle une restauration d'habitats et une remise en état de la voirie. La coupe stratigraphique de la voie sacrée, poursuivie en 1965 dans l'enclave militaire (parcelle 3407), a nettement montré l'exhaussement de la voie et son élargissement aux dépens des cases de l'îlot XI, dont les foyers et les trous de poteau avaient été enfouis sous une petite plare à fonction religieuse, de 8 mètres de largeur, devant le "sanctuaire des crânes ").

Le sol primitif de la voie, reconnaissable dans la partie sud aux alignements de pierre, avait été recouvert par une couche de rudus de quelque $0,30 \mathrm{~m}$ d'épaisseur, constitué par du matériau de remploi, fragments de stèles calcaires parfois posées de chant, de meules et de dolia, à l'exclusion de toute autre céramique (fig. 3). Au-dessus était tassée une couche de graviers, de galets et de sable (provenant d'une carrière de la banlieue aixoise située entre les hameaux du Pey Blane et des (iranettes), sur laquelle étaient quelques fragments de dolia et d'amphores. Or, les deux niveaux renfermaient des boulets de basalte, lancés par les balistes de l'assiégeant. Ln boulet du niveau supérieur a été trouvé entre deux crånes écrasés portant des traces d'enclouage (fig 4), comme ceux qui avaient été découverts précédemment dans la partie est du sanctuaire ${ }^{15}$. C'est pendant le court intervalle entre les deux sièges qu'auraient été faites des réfections hàtives en vue de réparer les dégâts de la première attaque. En ce cas, on peut supposer que les crânes de la voie sacrée n'ayant pas une grande ancienneté auraient été exposés après les combats de 125 et auraient appartenu à des ennemis, en l'occurrence des légionnaires romains, ce qui expliquerait l'acharnement des assiégeants contre le sanctuaire. La découverte de fers de pilum et de haste (fig. כ) lors de la reconnaissance du front ouest, où se trouvait l'entrée de la voie sacrée (dans le terrain militaire), est le signe du siège de la ville. Celle-ci avait été soumise à un violent bombardement de lithoboles, dont le plus grand nombre de boulets a été trouvé sur la voie sacrée, devant le sanctuaire et dans les quartiers limitrophes du

(11) Sur la localisation, v. note 10 supra.

(12) CESAR, B. (i., VII, 64, 8 .

(13) Gallia, XIV, 1956, p. 220, fig. 3.

(14) Ibid., XII, 1954, p. 286, fig 1.

(15) Ibid., p. 292 et Le sanchuare aus "esprils" d'Entremonl, dans Cahiers ligures de prehistoire el d'archéologie, 4, 1955, p. 38. 


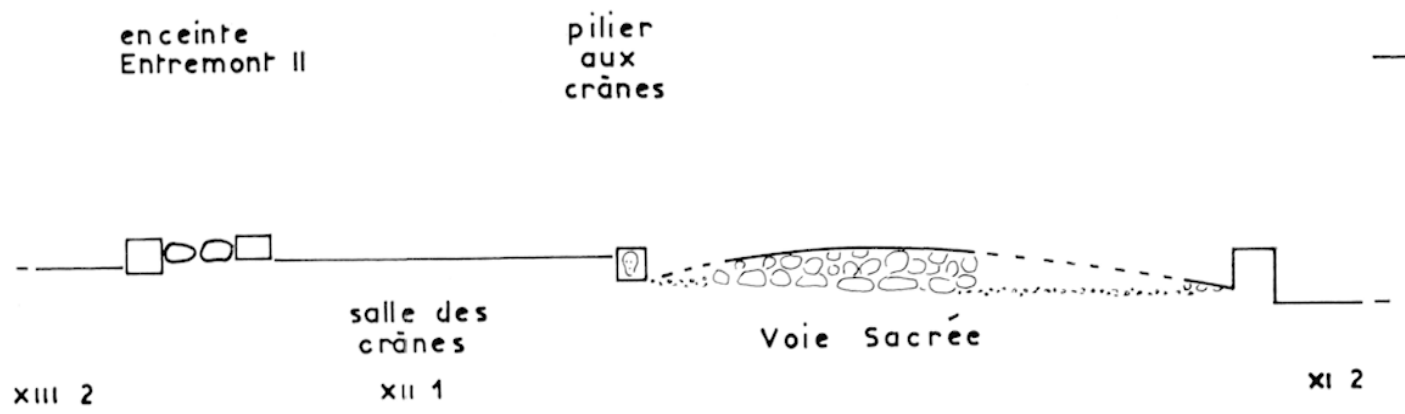

$2 \mathrm{~m}$.

3 Coupe de la voie sacré du nord au sud, montrant liexhaussement du niveau entre les deux sieges.

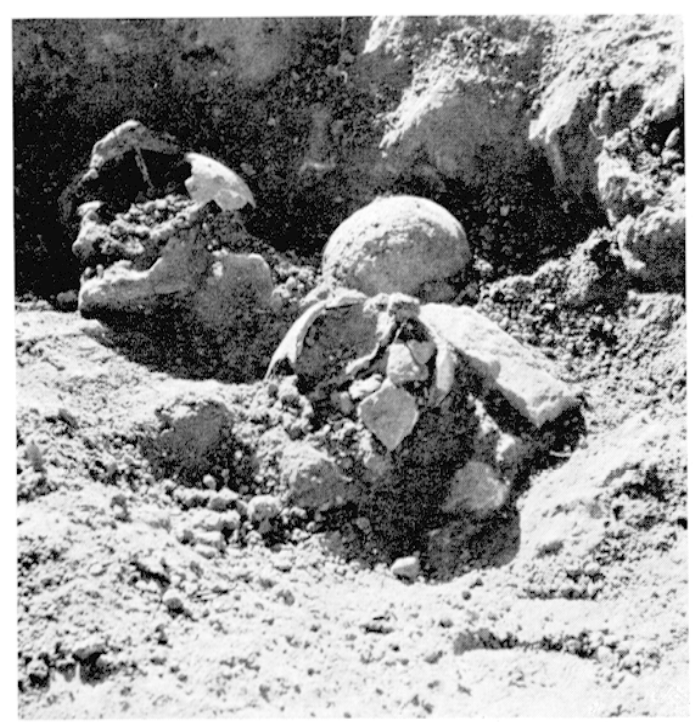

1 Crànes avec trace d'enclouage écrasés de part et d'autre d'un boulet de basalte,

au niveau supérieur de la voie sacrée.
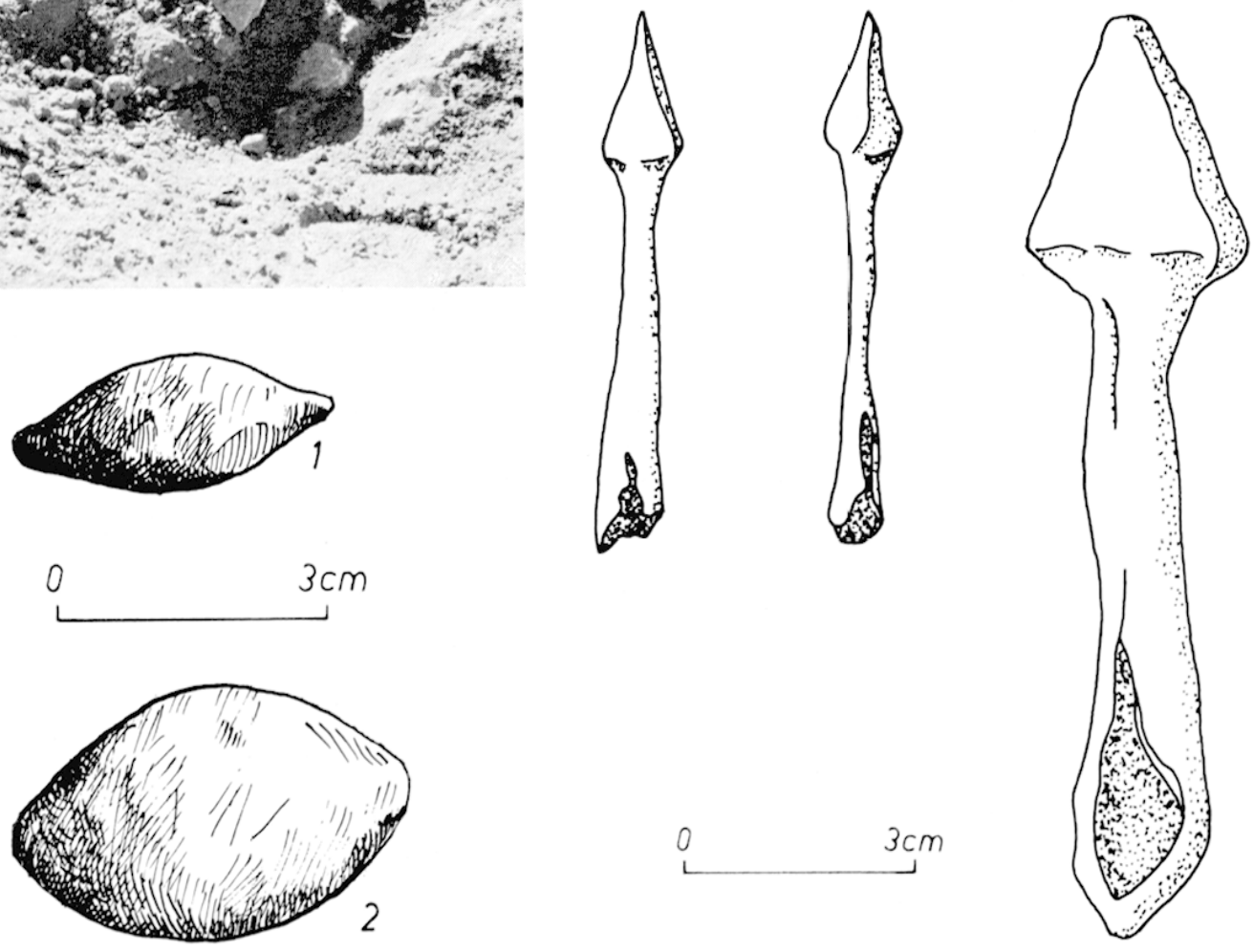

6 Balles de fronde : 1 , en plomb : 2 , en terre cuite.

5 Fers de javelot et de pilum. 
rempart nord (rue IX, îlots VIII et I). Ces boulets, la plupart en basalte, ont un diamètre très inégal (basalte : 0,128 à 0,191 ; pierre : 0,15 à $0,168 \mathrm{~m}$ ) et un poids allant de 3,730 à $8,335 \mathrm{~kg}$, analogues à ceux de Roquepertuse mais légèrement inférieurs à ceux du siège de Marseille et à ceux de Saint-Blaise (poids des boulets de pierre : 3,500 à $18,200 \mathrm{~kg})^{16}$.

Quelles étaient les armes des défenseurs ? Des flèches, des javelots, des dagues en fer, et surtout de très nombreuses balles de fronde en terre cuite (long. : 0,045$)$ (fig. 6, 2); on a retrouvé deux groupes de 41 et 25 balles dans la rue IX. La case VIII. 5, encore incomplètement fouillée, possédant au moins trois jarres à eau, paraît avoir été un atelier de fabrication de ces balles (on y a retrouré une masse d'argile délitée). On notera la rareté des balles de fronde en plomb, de forme oblongue, effilée aux deux extrémités, faites d'une feuille de plomb repliée (long. : 0 , 035) (fig. 6, 1), comparativement à leur abondance à Saint-Blaise et à l'oppidum de Pierredon à Eguilles.

La ville fut pillée avant d'être abandonnée : les rues étaient jonchées de dolia et de poteries provenant des cases (rues I, IX, XVII etc...) et la plupart des cachettes de monnaies, creusées dans le sol à même la terre ou dans le banc de rocher, avaient été violées. Deux d'entre elles, cependant, ont été retrouvées intactes dans la ville haute et deux autres dans la ville basse, à l'angle des pièces.

\section{LES DEUX REMPARTS}

La première fouille, orientée sur le front ouest, en raison de la découverte de fragments de statuaire en 1943, avait mis au jour la voie sacrée qui gravit la colline en direction générale est-ouest. Son tracé, à l'intérieur de l'enceinte, est marqué par des ornières creusées dans le sol rocheux et le cailloutis, distantes d'axe en axe de 1,35 m environ. Elle s'infléchit vers le nord et, après son passage dans le terrain militaire, a été retrouvée parallèlement et à l'extéricur de l'enceinte d'Entremont II. Ce rempart, assis sur de grands blocs formant saillie, reposant directement sur le sol, sans fondation, a été dégagé sur 60 mètres de long sur le front nord. Recouvert d'une couche de terre peu épaisse, il a été arasé, après l'abandon de l'oppidum, à un niveau inférieur aux murs des cases d'Entremont II qui lui sont adossées - arasement qui ne peut s'expliquer que par l'enlèvement des grands blocs dépassant le niveau du sol, en vue de leur remploi. Seules subsistent une ou deux assises au-dessus des substructions, dans la tour de l'ouest (fig. 10 et 11 ).

I)'une largeur moyenne de $1,50 \mathrm{~m}$, ce rempart était défendu par des tours carrées sans remplissage, distantes de 19 mètres environ, soit 60 pieds, entre lesquelles seront aménagées de grandes cases à la période suivante. Le matériau, qui comprend en remploi quelques fragments de stèles aniconiques en calcaire et une autre ornée d'un signe en "épi de blé " en pierre de Bibemus (XII. 6), provenant du sanctuaire primitif (Entremont I), est emprunté au banc oligocène du plateau. L'angle nord-est, dépourvu de tour, en porte-àfaux, repose sur un mur d'ancrage, disposé de biais, qui sert de fondation au rempart. La face est, en surplomb sur la ville basse, reconnaissable à un alignement de gros blocs formant sa base (certains ont $1,70 \mathrm{~m}$ de longueur), est à fleur de terre; elle marque la délimitation cadastrale.

(16) H. Roluand, Fouilles de Saint-Blaise, IIIe supplément à Gallia, 1951, p. 131. 


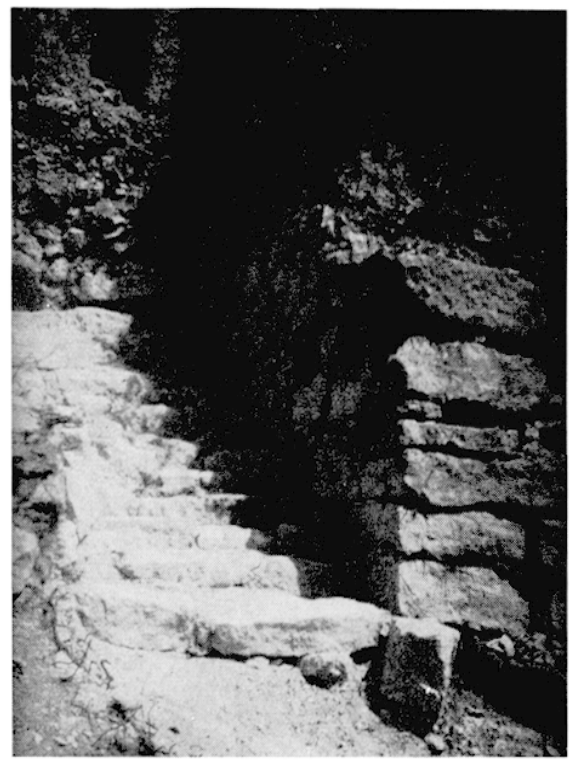

7 Poterne de l'enceinte d'Entremont III sur la face sud.

9 Face ouest de la tour 3 et départ de la courtine, $\rightarrow$ montrant les assises de substruction faites en tranchée.

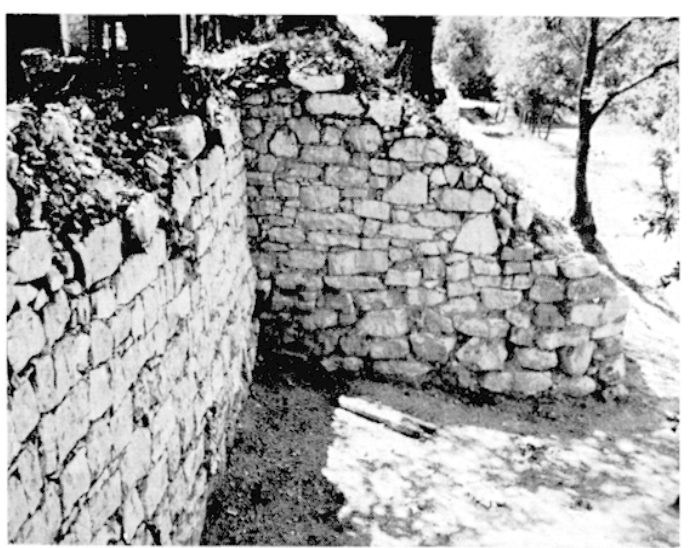

* Enceinte d'entremont III. Face est de la tour 3 a angle curviligne, apris restauration.

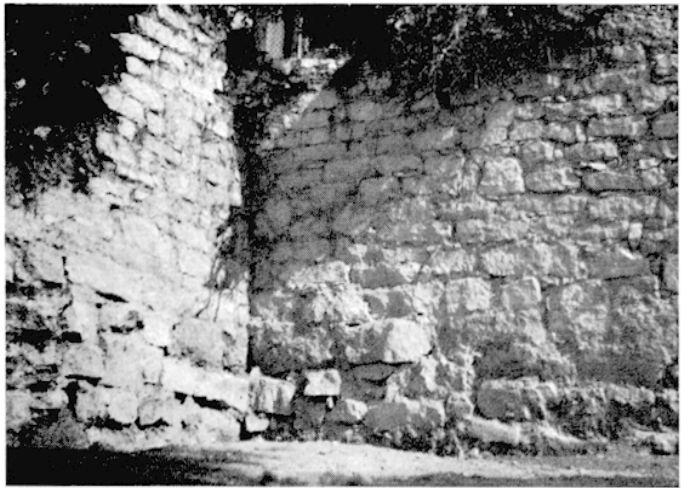

Ainsi, dans l'état actuel de la fouille, la ville haute (Entremont II) apparait comme un quartier complètement séparé de la ville basse, longé par la voie sacrée qui lui est extérieure. - sans qu'aient été encore reconnues de communications avec cette voie et la ville basse. Selon la coutume des oppida, ni ce rempart, ni celui d'Entremont III n'avaient de lice intérieure pour la défense, les cases étant adossées au parement de l'enceinte.

L'enceinte d'Entremont III, qui barre le plateau au nord sur 380 mètres environ de longueur, a été dégagée ou reconnue sur 130 mètres à partir de l'ouest à la limite de la clôture militaire et à l'angle nord-est. D'une épaisseur de $3,15 \mathrm{~m}$, clle présente un appareil plus régulier et plus soigné que le rempart d'Entremont II, sans remploi de stèles. Les blocs sont calés par de petites pierres plates. L'appareil du parement intérieur, plus petit, est analogue à celui des murs des cases qui s'appuient à l'enceinte. Aucune porte dommant accès à la ville basse n'a encore été reconnue ; par contre, une poterne, avec escalier de descente, large de $1,10 \mathrm{~m}$, a été mise au jour sur le front sud, vers la plaine où se trouvaient les points d'eau (fig. 7); mais le rempart a été emporté par la poussée des terres qui avaient enseveli la poterne sous une masse de 5 mètres de déblais.

Le dégagement partiel du rempart nord a permis de reconnaitre dix tours distantes de 19 mètres environ, comme celles d'Entremont II. Enfermant un massif de blocage, elles se différencient des premières par le profil curviligne des angles destiné (fig. 8 ) à résister aux coups de bélier. C'est là une technique savante, qui se retrouve à l'oppidum de Pierredon à Eguilles. Sa hauteur atteint près de 4 mètres en certains points, mais sa construction était 


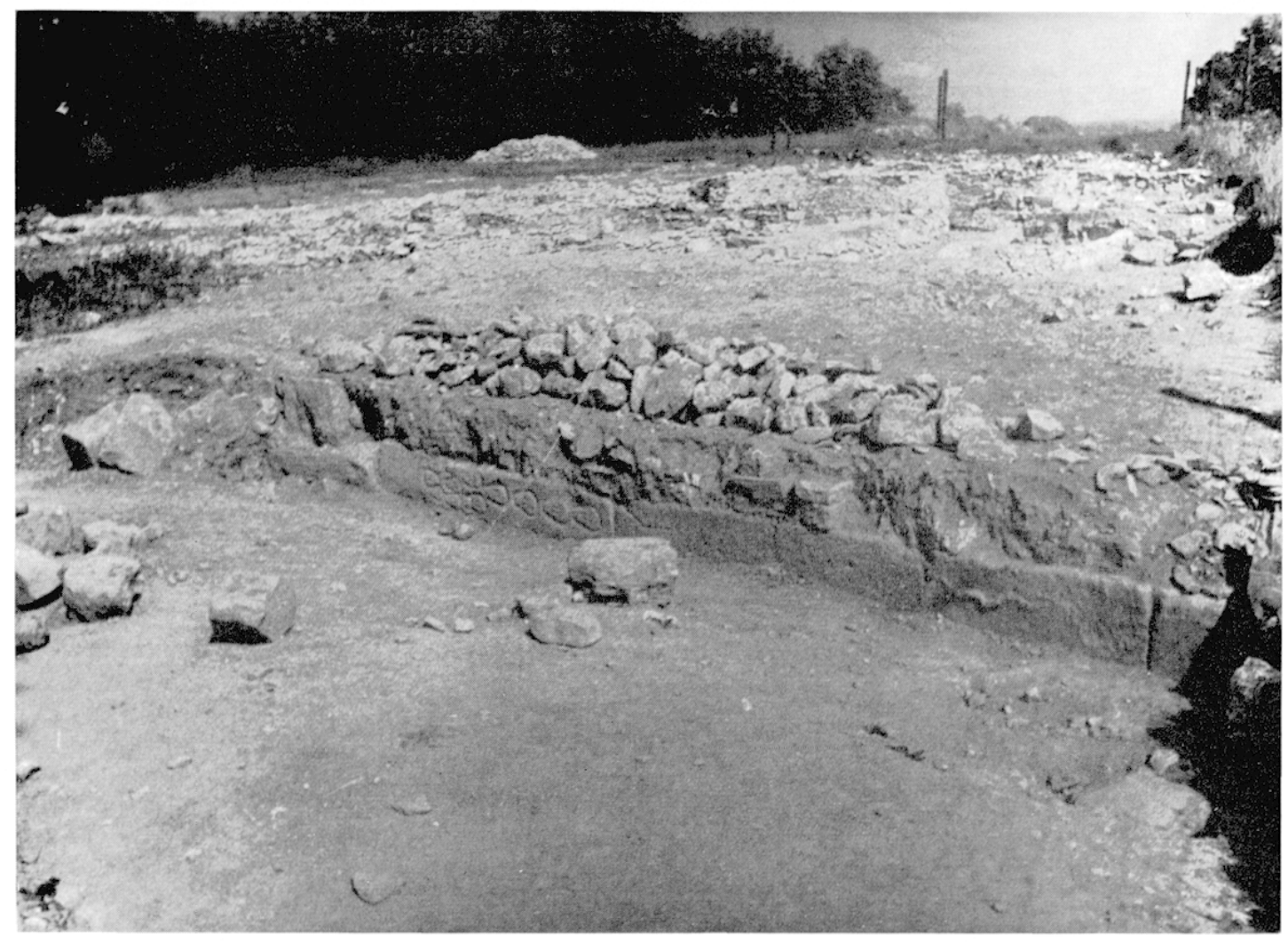

10 Le sanctuaire aux crànes. Le stylobate est constitué par des piliers d'Entremont I décorés de douze têtes sans bouche et d'un serpent). A gauche, bloc d'angle de la tour 2 du rempart d'Entremont II avant le degagement.

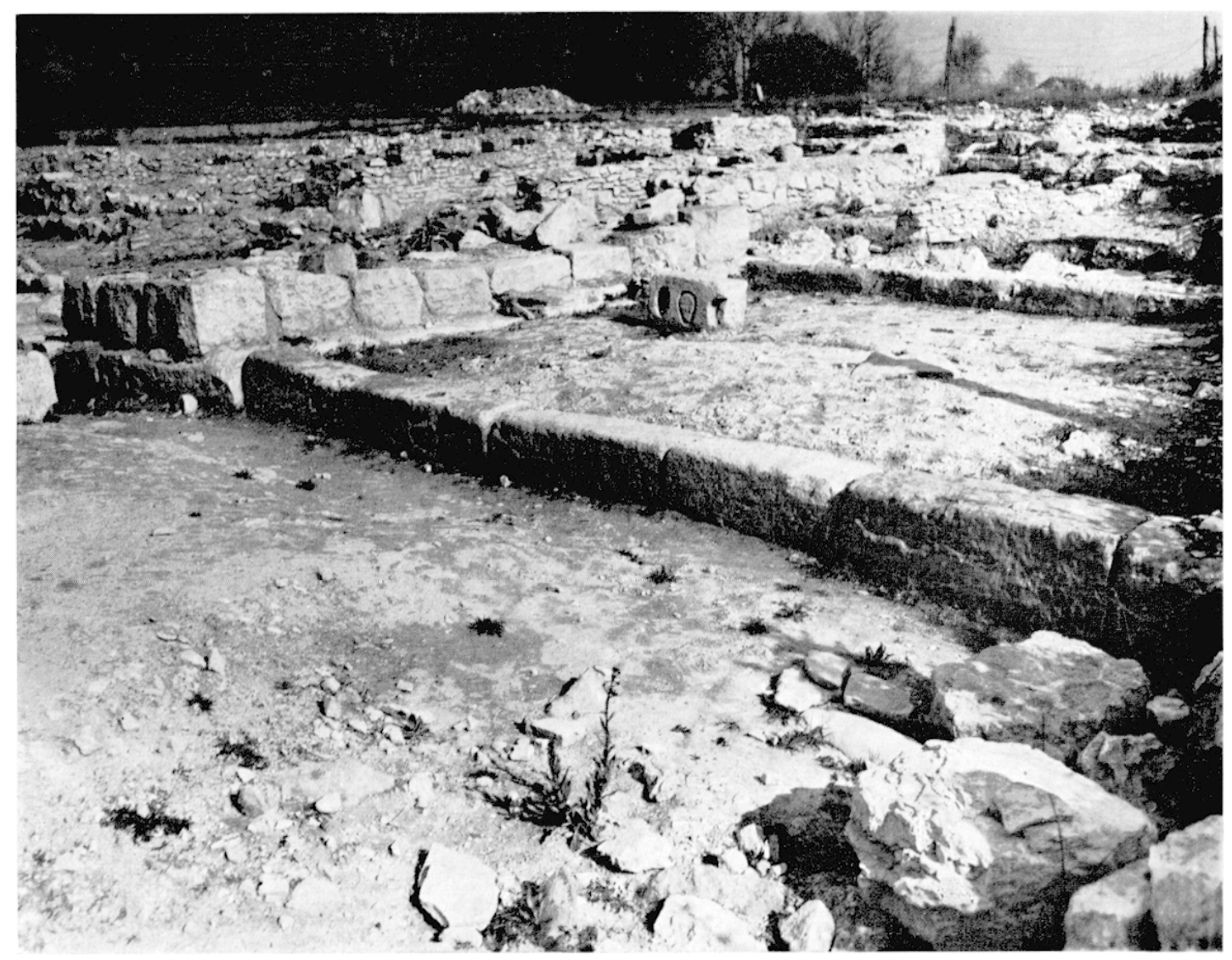

11 Stylobate du sanctuaire aux crànes ut angle n.-0. de la tour 2. 
primitive et peu apte à la défense : la base du rempart, construite en tranchée, suit la dénivellation du terrain qui n'avait pas été aplani, de telle sorte que les assises de fondation, faites de blocs grossièrement équarris et saillants, présentent un aspect irrégulier (fig. 9), en contraste avec la régularité relative des assises supérieures. Cependant, sa technique de construction est évoluée si on la compare à celle des oppida gaulois : à l'angle est de chacune des tours avait été ménagée une canalisation pour l'évacuation des eaux pluviales, dont l'ouverture, à la base du rempart, était couverte par une grande dalle formant linteau.

Sur la face ouest du plateau, l'escarpement rocheux servait d'assise au rempart, qui a été masqué par les "restanques" de pierre sèche construites pour retenir les terres de culture. Dans une enclave du terrain militaire (îlots II et III), l'enceinte est apparue bouleversée par les travaux agricoles. La base d'un mur épais seulement de 1, 50 m a été dégagée. Faut-il attribuer à cette enceinte quelques grands blocs de substruction (long. : $0,90$; larg. : 0,50$)^{17}$ ? A ce niveau a été recueilli un denier de C. Minucius Augurinus, monétaire vers 129 (poids : 3, $86 \mathrm{gr}$ ). Au droit, tête casquée de Rome à droite ; au revers, colonne ionique de forme tronconique entre deux logalis

Des deux enceintes, il apparaît que celle d'Entremont III est la plus récente; c'est elle qui fit front aux assiégeants, ainsi que le montre le nombre de boulets de lithoboles et de balles de fronde trouvés à proximité du rempart, à l'intérieur de la ville. L'enceinte d'Entremont II, si elle formait encore une clôture, avait perdu son rôle défensif : des cases avaient été aménagées dans l'intervalle des tours ; la plus grande, le "sanctuaire aux crânes " (XII. I), qui devait occuper tout le front entre les deux tours de l'ouest, c'està-dire 18 à 19 mètres, a été dégagée sur 16 mètres de longucur. Sa façade nord sur la voie est constituée par un stylobate (fig. 10 et 11), dont ont été mis au jour deux piliers décorés (l'un de 12 têtes coupées, l'autre d'un serpent en relief), qui avaient été remployés; d'autres en pierre de Bibemus et en calcaire oligocène, sans décoration, épais de $0,40 \mathrm{~m}$ environ, portent sur leur face supérieure de remploi des traces d'encastrement de supports (sans doute de pierre, plutôt que de bois, en raison de leurs dimensions et du peu de profondeur des cavités : $0,03 \mathrm{~m}$ environ), groupés deux par deux, encore que la concordance des écarts ne soit pas rigoureuse. Sur la face opposéc. plaqué contre le rempart d'Entremont II (fig. 12), le stylobate est également constitué par des piliers remployés, d'une épaisseur moyenne de $0,30 \mathrm{~m}$, dont l'avant-dernier présente à son sommet un alvéole céphaliforme de $0,27 \mathrm{~m}$ de hauteur sur $0,16 \mathrm{~m}$ dans sa plus grande largeur. Dans l'axe médian de la salle, qui avait 6 mètres environ de largeur, une série de blocs enfoncés en terre affleurent au sol. Ils étaient destinés à soutenir la toiture ou l'étage d'une salle hypostyle ouverte

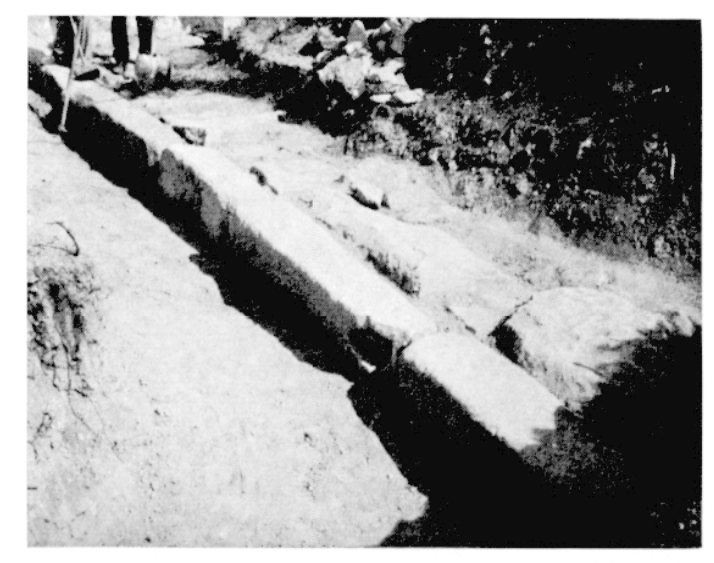

12 Assise inférieure de la façade postérieure du sanctuaire, adossée au rempart d'Entremont II. Pilier à alvéole céphaliforme en remploi.

(17) Gallia, VIII, 1950, p. 118 ; XVI, 1958, p. 414 ; XVIII, 1960, p. 291.

(18) E. Babelon, Monnaies de la République romaine, II, p. 229, 3. 


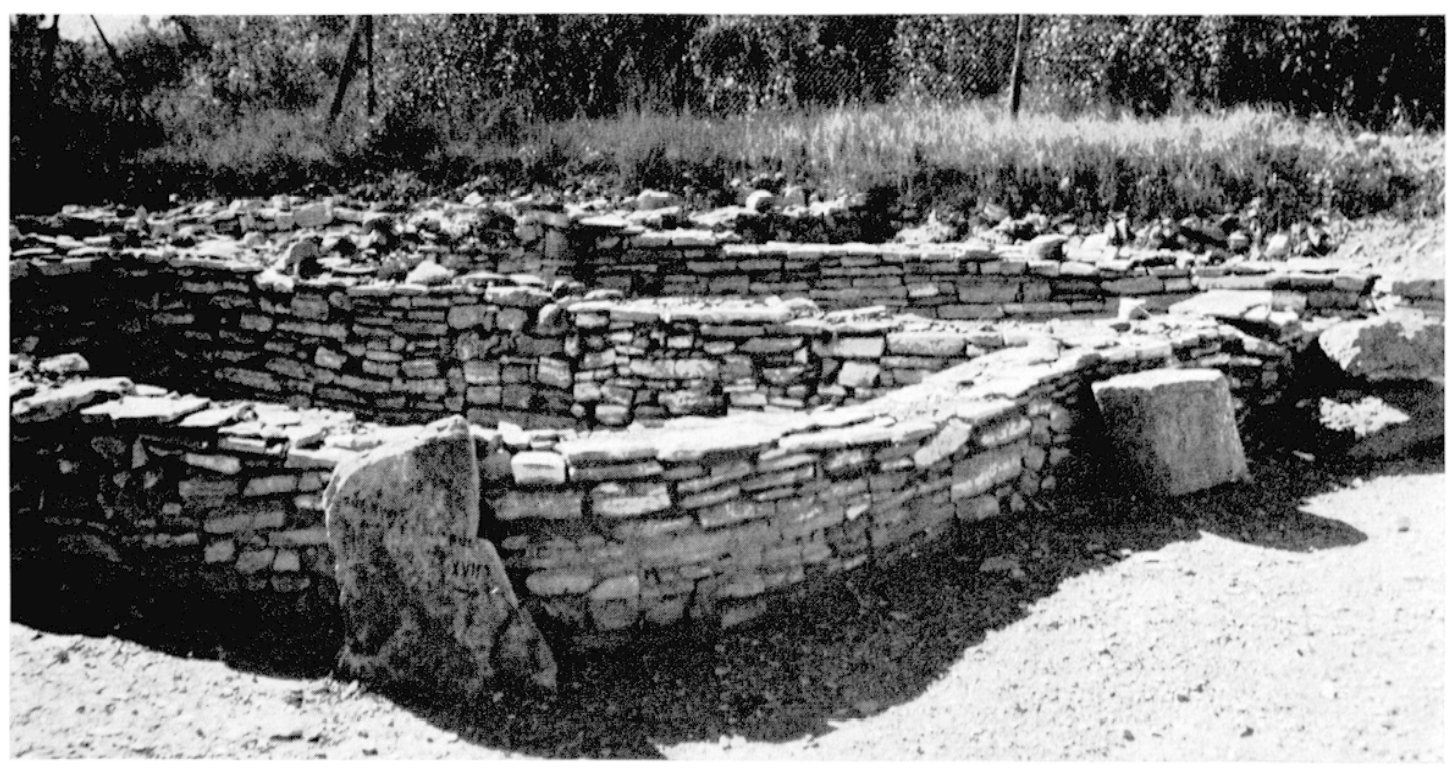

13 Case a angle curviligne et chasse-roue de l'îlot $\mathrm{XX}$ (ville haute), à l'intersection des rues XVII at XVIII Noter l'association de l'appareil en dalles plates do gypse et de moellons irréguliers.

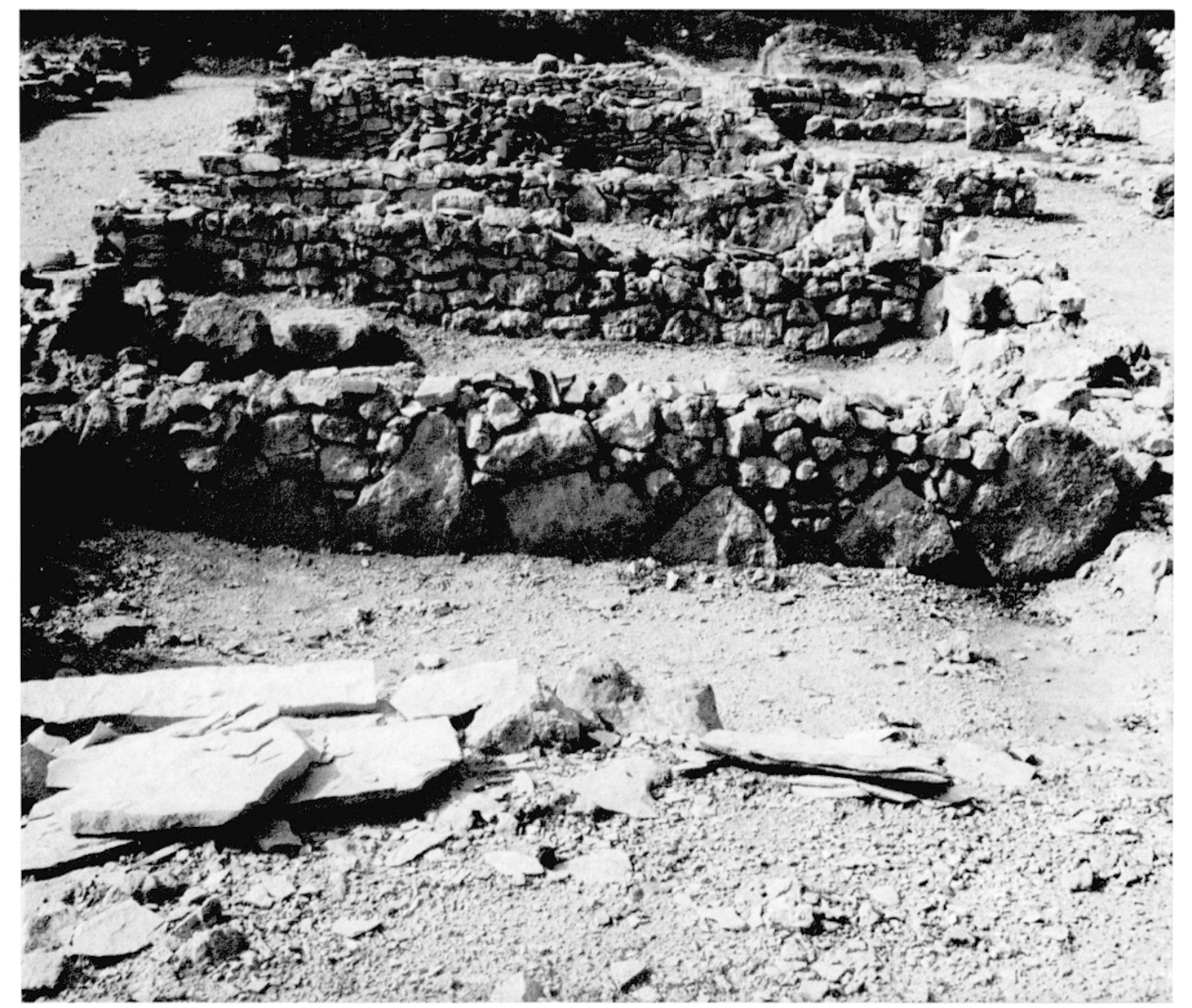

14 Alignement de cases á une piece de l'ìlot XVI donnant sur la rue XVII ville haute. Au premier plan, mur en moellons irreguliers reposant sur des dalles de chant iorthostates). 
sur la voie sacrée : des fragments de mosaïque en opus signinum friable avaient été découverts, en position inclinée, en $1954^{19}$.

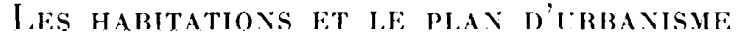

Le plan d'urbanisme des deux quartiers révèle une orientation différente. Le quadrillage des rues et des cases de la ville haute a été tracé selon l'axe de l'enceinte d'Entremont II, tandis que celui de la ville basse se raccorde en éventail avec cette enceinte : la "voie sacrée " et la rue XI délimitent un îlot trapézoïdal et la rue I est parallèle à l'enceinte d'Entremont III. Lne tranchée de prospection (T. XVI), faite en $1946^{20}$ dans le secteur est de la ville basse (parcelle 3412), montre la mềme orientation, parallèle à l'enceinte d'Entremont III. On doit donc en conclure que le plan d'urbanisme d'Entremont III a été suivi sur tout le front nord et qu'il est contemporain de la construction de l'enceinte.

Le plan général, malgré la différence d'orientation des deux quartiers, est un intéressant exemple d'urbanisme : il suit une tradition grecque ou étrusque qui a été reconnue dans le comptoir massaliète d'olbia (Hyères) ${ }^{21}$. Les îlots d'habitation sont séparés par des rues de $2,50 \mathrm{~m}$ a $4 \mathrm{~m}$ de largeur, qui se coupent à angle droit. Le même mode de construction caractérise les deux quartiers, dont le dernier état semble contemporain, les murs ayant été relevés à différentes reprises, en raison de leur fragilité.

Dans l'un et l'autre quartiers, les angles des cases sont curvilignes (fig. 13), comme ceux des tours de l'enceinte d'Entremont III, et parfois protégés par un garde-fou fait d'une grande dalle de chant ou d'un fragment de dalle de pressoir (carrefour des rues IX et XI). Les portes sont défendues par d'épais contreforts (XV. 6; VIII. 4; I. 7). On note l'emploi de techniques identiques dans les deux quartiers : base des murs faite de grandes dalles trapézoïdales posées de chant ou orthostates (fig. 14) (XVI. 3, 4 et 5 ; X. 6 et 11) ou de grosses pierres allongées (XV. 3,4). Mais, en tenant compte des réfections, l'appareillage des murs d'Entremont II, qui utilise couramment les dalles plates de gypse, débitées régulièrement, contraste avec l'opus incerlum de grossiers moellons d'Entremont III. La réfection d'une case de l'îlot XV. 6, agrandie par la réunion de deux cases étroites, modification qui nécessita le soutien d'un pilier de bois pour étayer la toiture, montre la différence d'appareil entre ce mur et les autres murs de l'îlot.

La restauration des murs est confirmée par la substitution de la pierre aux briques d'argile crue, sans qu'il soit possible de conclure à la généralisation de cet emploi. Des briques reposant sur un socle de pierre et le bâti en argile d'un poteau étaient en place dans l'îlot III de l'enclave ouest ${ }^{22}$. Des fragments de briques écrasées (larg. 0, 12), mêlés à des fragments de dolia, ont en effet été retrouvés dans le comblement des cases dont le sol avait été légèrement exhaussé, recouvrant d'anciens foyers (XI. 3, 9 et X. 4). Cet exhaussement, irrégulier et de peu d'épaisseur, ne peut être un indice de datation, les fragments de céramique trouvés aux deux niveaux étant de même époque.

(19) Gallia, XII, 1954, p. 291.

(20) Plan hors texte, Gallia, 1,1947, p. $x 1$.

(21) Ibid., XII, 1954, p. 6, fig. :2.

(22) Ibid., XVI, 195x, p. 114, fig. 4 et 5. Plan, ibid., NiIII, 1960, p. 291. 


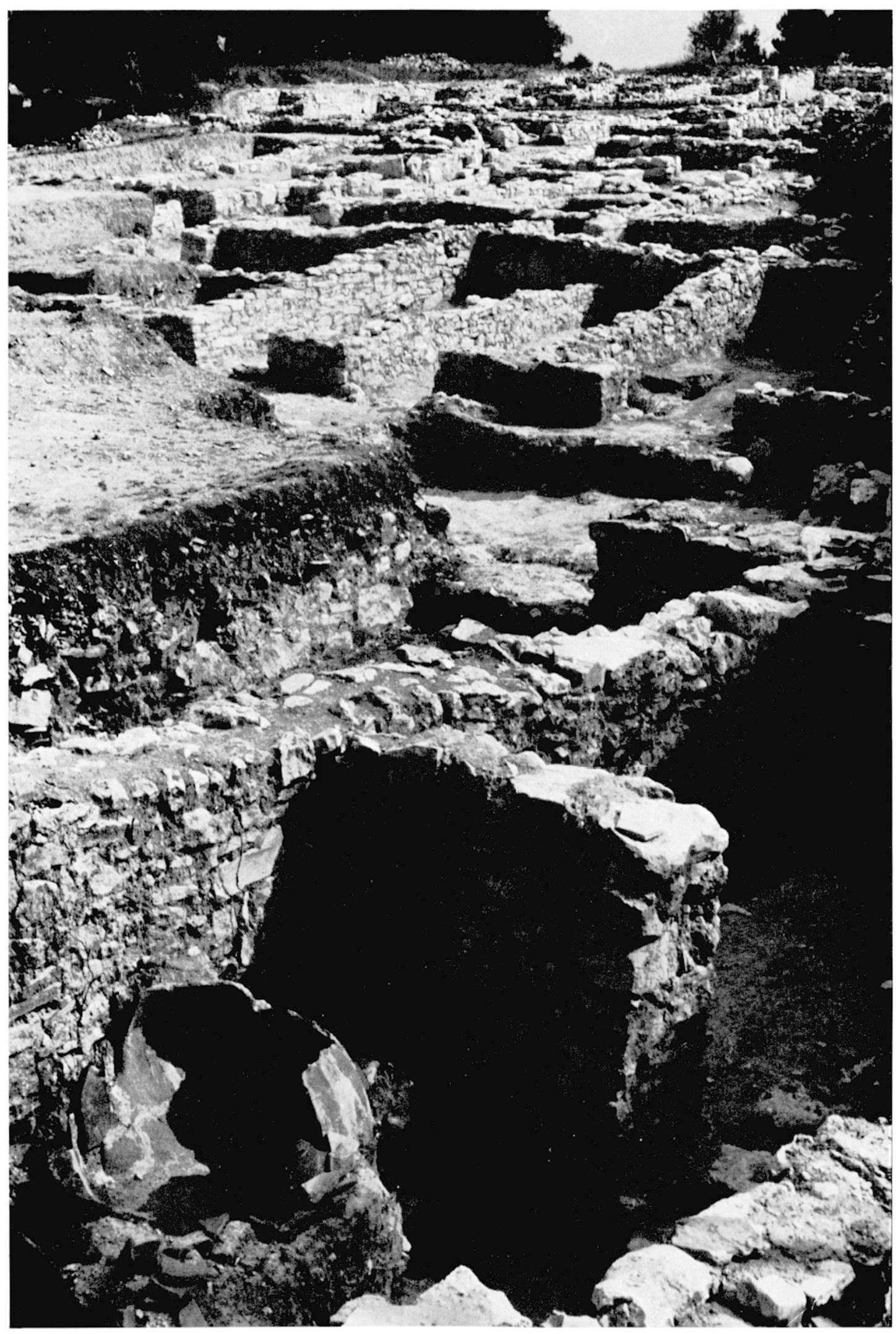

15 Vue générale du n. au s. prise de la tour 3 de l'enceintr d'Entremont Il I. Au premier plan, case 2 de l'îlot 1 et sol primitif de la rue 1 ; les dolia renversés reposent sur le second niveau de la case au-dessus de la canalisation comblée. Au sud de la rue, les cases 10 (foyer) et 6-11 avee cloison interieure, de l'ilot $X$, adossées a un mur a orthostatus. 
Le plan des cases de ces deux quartiers présente cependant, malgré l'exiguïté du secteur légagé, une différence: tandis que les rues XVII et XIX d'Entremont II renferment d'étroites cases à une seule pièce, en général dépourvues de foyer, dont la forme allongée et étroite (5) a 8 mòtres de longueur sur $2,50 \mathrm{~m}$ à $3,50 \mathrm{~m}$ de largeur) se retrouve dans les cases de l'enclave ouest, à fonction artisanale, comme l'indique la présence d'areae de pressoir et re faucilles en fer, mais toutes pourvues d'un foyer, les habitations d'Entremont III comprennent deux ou plusieurs pièces, avec magasin de dolia, pressoir à huile elc. On notera en particulier la superficie des habitations comprises entre les rues I et IX (îlots I et $X$ ), dont la partie occidentale est encore engagée dans le terrain militaire (figr. 15). Lne grande salle de l'îlot XI sera divisée en deux par une mince cloison de 0,30 m d'épaisseur (XI. 3 et 10). La même subdivision a été faite dans une case de l'îlot X, dont une pièce avail conservé en place, fiché en terre, le piquet de fer avec anneau pour l'atlache d'un chien (X.12).

L'une de ces habitations (ilot $\mathrm{X}$ ), ouvrant sur la rue par un vestibule avec banquette de pierre adossée au mur, comprend plusieurs salles prenant jour sur une petite cour (fig. 16), à la façon de la maison méditerranéenne de plan hellénistique. La cour donnait accès à un magrasin sans éclairage et à une huilerie, dont la dalle ou area (long. : 1, 70 m) était en place, ćtablie a prese un léger exhaussement du sol (fragments de dolia et de céramique campanienne et indigène). En façade, la pièce 8 . sans communication avec le restant de l'îlot et ouvrant directement sur la rue, était peut-être une boutique, ainsi que le montre le mobilier abondant et varié qui y a été trouvé : débris d'amphores, dont un avec timbre PI.M.R., céramique massaliète tardive à bandes peintes et indigène, anneaux de bronze, agrafes de réparation de dolia en plomb et débris de dolia, fusaïoles, passe-guide en bronze en forme d'oméga terminé par deux têtes de canidés (fig. 22), elc.

Les seuils, rudimentaires, sont constitués de dalles plates, sauf dans le quartier des fours de potier de la voie sacrée, où l'exhaussement de la voie a entraîné le relèvement des seuils primitifs et leur doublement par une dalle avec arrêt pour la fermeture du vantail et crapaudine (XI. 4) 23. C'est également l'exhaussement de la rue IX qui a rendu nécessaire le doublement du seuil primitif de la boutique (X. 8) par une dalle comportant une butée pour la fermelure de la porte.

L'exhaussement de la rue I, parallèle au rempart, à la suite d'un incendie, dont la couche de cendres est visible à l'ouest sur $0,50 \mathrm{~m}$ de hauteur, avait amené l'obturation de la canalisation débouchant à l'est de la tour 3. Les cases de cet îlot, suivant la dénivellation du terrain, dans leur état primitif, étaient étagées : celles qui étaient situées au sud de la rue, établies sur le rocher en surplomb de celle-ci, ont conservé leur seuil primitif auquel on accédait par plusieurs degrés, de telle sorte qu'elles se sont trouvées, ainsi que leur seuil, de plain pied avec la nouvelle voie, tandis que les cases situées entre la rue et le rempart furent exhaussées de la même hauteur $(0,50 / 0,75 \mathrm{~m})$, ce qui avait entraîné la suppression de la canalisation et l'établissement d'un nouveau sol marqué par la présence de trois dolia restés en place, au-dessus de l'ancienne ('analisation hors d'usage (fig. 15). 
Le matériau de construction des murs, dont l'épaisseur ne dépasse pas 0, 50) $\mathrm{m}$ (parfois même $0,30 \mathrm{~m}$ dans les cloisons), est en pierre sèche sans autre liant qu'un mortier de terre. La paroi intérieure de certains murs était enduite d'argile. La case aménagée entre deux tours de l'enceinte d'Entremont II (XII. 4), reconstruite après le premier siège, ainsi que semble le montrer la présence d'un boulet dans le remblaiement, avait un parement interne plus soigné : à des plaques d'argile cramponnées par des clous de fer à tête convexe étaient associés des parements d'argile stuquée, décorés en relief de cannelures ou triglyphes ${ }^{24}$.

Le sol était en terre battue, les creux de l'assise rocheuse étant aplanis par de la pierraille ou des dalles plates irrégulières, en certaines parties seulement de la pièce. La toiture, qui ignore la tuile (rare même à Marseille à l'époque romaine, si l'on en croit Vitruve), était sans doute couverte en terrasse faite de clayonnage de bois et de torchis, peut-être de peaux de bêtes. Elle était, dans les plus grandes cases, étayée par un poteau de bois reposant sur un bloc de pierre.

Le foyer d'argile était adossé au mur, souvent à l'angle de façade de la pièce pour assurer l'évacuation de la fumée par une ouverture, mais parfois au milieu de la pièce. Construit sur un bâti de pierraille (XI. 3) ou de fragrments de dolia (X. 3; XI. 9), il est particulièrement bien conservé dans les cases de l'îlot I en cours de fouille (cases 10.6, II). Aucun de ces foyers ne porte une décoration analogue à celle qui avait éte trouvée dans une case de l'îlot II bordant la voie sacrée; orné de rouelles juxtaposées, imprimées avec un roseau, malgré son caractère primitif, il suivait la tradition des autels-foyers, a la mode grecque, ornés de méandres ou de svastikas (La Roque de Fabrègue, Substantion, Lattes, Cayla-de-Mailhac en Languedoc).

Les cases avaient, toutes, un ou plusieurs dolia encastrés dans le sol pour les réserves d'eau ou de grains, et contenaient des débris d'amphores vinaires. Les dolia étaient parfois groupés dans de véritables magasins occupant le fond de la maison, sans lumière (cases adossées au rempart : I. 2 et 5 , peut-être X. 3).

Chaque habitation paraît avoir eu sa cachette ou trésor. creusée dans la terre ou le, rocher, recouverte d'une dalle plate, généralement à l'angle et au fond de la pièce. Deux de ces trésors ont été trouvés dans la ville haute : la reconnaissance du rempart oriental séparant la ville haute de la ville basse (T. XXIII) a amené la découverte d'un trésor de 1435 oboles de Marseille à la rouelle, à tête d'Apollon à gauche, certaines portant gravée sur la joue la signature MAP ou MA ; clles étaient contenues dans un pot de terre jaunâtre délité; moins anciennes que celles du trésor de Lattes, elles remontent au début du ${ }_{11}{ }^{\mathrm{e}}$ siècle ${ }^{25}$ av. J.-C:

Dans le même quartier (case XX. 3), un silo de un mètre de hauteur et $0,60 \mathrm{~m}$ de diamètre, creusé au fond et au coin de la case dans le rocher et revêtu de plaques re calcaire cramponnées avec des clous, appartenant au premier habitat dont les murs sans fondation avaient été remontés à $0,15 \mathrm{~m}$ plus haut, contenait, au-dessous d'une masse de fer et de pointes de javelots en fer, 100 oboles de Marseille à tête à gauche, 2 drachmes au lion et

24) Gallia, XVI, 1958, p. 414, fig. 3

25) Ibid., V, 1947, p. 83; H. Rol.tavn, Troucaille d Entremont, dans Revue numismalique, 2, $1959-1960$, p. 37 : R. Majtrif, J. Arxal, II. Prades, Le trésor de Lalles, dans Ogam, XVIII, 1966, p. 280. 


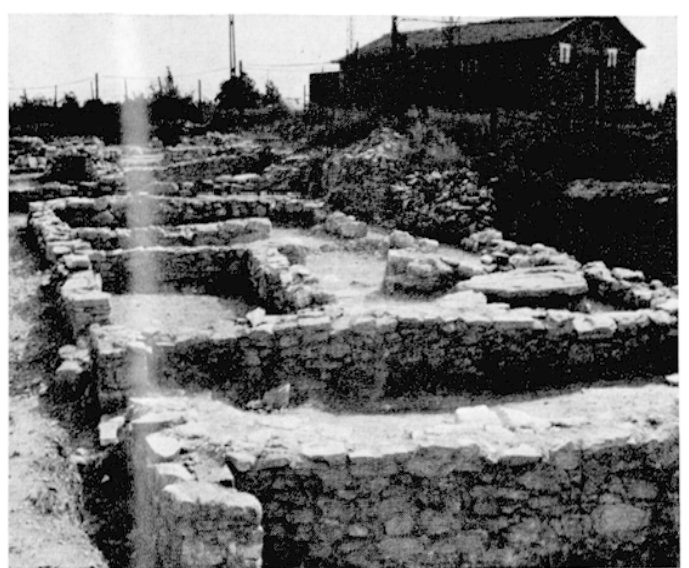

16 Habitation a plusieurs pieces de l'îlot $X$ ouvrant sur la rue IX. Pressoir en place dans l'huilerie. An premier plan, case 9 avec son dolium en place.

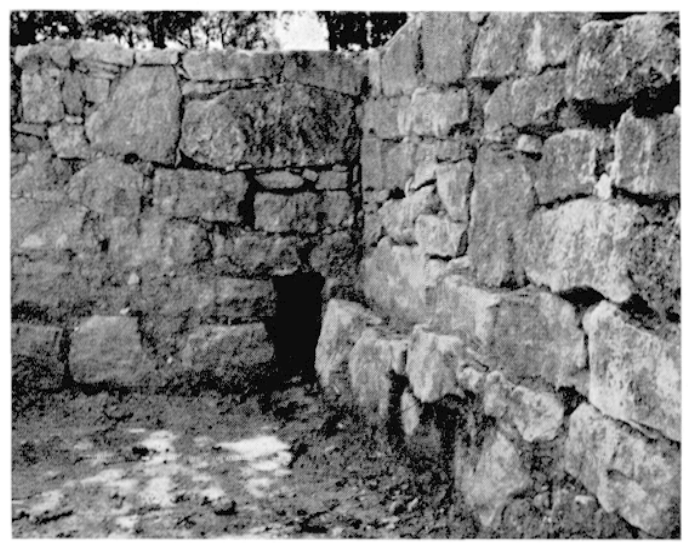

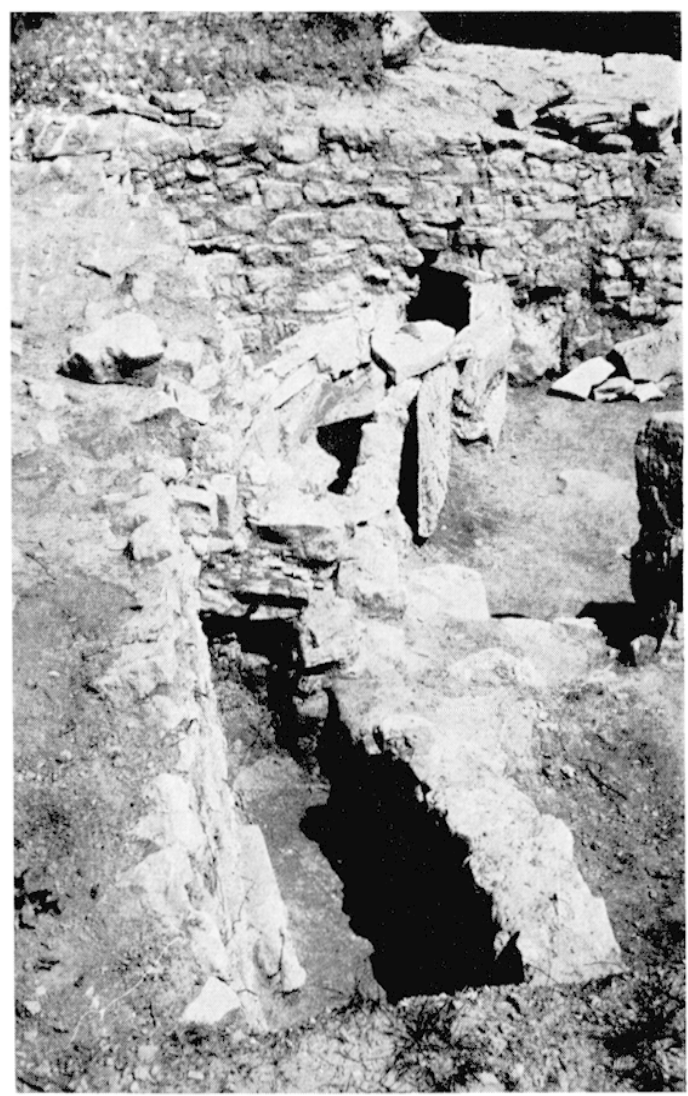

18 Canalisation pour l'evacuation des eaux de pluie dans les cases 1 et 3 de l'ilot VB 1 adossé à la tour 5 .

\& 17 Orifice de sortie de la canalisation de l'ìlot 1 , débouchant au ras de la tour 3.

3 deniers de la République, d'un type sans monogramme des environs de 200 (1 quinaire au type de la déesse Rome a droite, et au revers les Dioscures avec légende ROMA et fer de lance vertical, et 2 victoriats à tête de Jupiter et au revers Victoire couronnant un trophée, l'un avec la truie au pied de la Victoire et le Génie de Rome (240-197)

Deux cachettes inviolées ont également été trouvées dans la ville basse, l'une de 110 oboles (X. 8), l'autre de 27 drachmes (X. 9).

\section{La volrie. L'évacuation des ealx de plite}

Le système d'évacuation des eaux de pluie donne un intérêt lout particulier à l'urbanisme de la ville. La déclivité générale du plateau qui ramassait les eaux au pied du rempart d'Entremont III avait fait prévoir, lors de la construction de cette enceinte, des canalisations qui débouchaient à l'angle est de chacune des tours dégagées à ce jour, parfois dans des puisards au niveau des fondations. Ces canalisations étaient soigneusement construites de grandes dalles (de fond posées de chant), la face extérieure brute, et couvertes de dalles. 
Dans l'îlot I, la canalisation qui débouche à l'est de la tour 3 par un large orifice couvert d'un linteau $(0,60$ sur $0,40 \mathrm{~m}$ ) (fig. 17) recueillait les eaux de la rue par une rigole creusée à mème la terre, ce qui avait nécessité le doublement du mur de façade $(1,20 \mathrm{~m})$ pour éviter la dégradation produite par le passage de l'eau. Elle avait elle-même succédé à une petite rigole creusée à même le sol de la case $(0,06$ sur $0,10 \mathrm{~m})$. Mais, à la suite d'un incendie, nous l'avons vu, l'exhaussement du sol de la rue et de la case avait entraîné le comblement de la canalisation et l'obturation, par des pierres de petit format, de l'orifice de sortie. La canalisation de la tour 4 débouchait par un orifice haut de 0,75 et large de $0,50 \mathrm{~m}$, soigneusement ménagé sous un linteau; le plafond à l'intérieur du rempart est formé de grandes dalles mesurant quelque $0,50 \mathrm{~m}$ de largeur; elle recevait les eaux de deux conduits adossés au mur de séparation de deux cases contiguës (I. 3 et I. 4), qui se rejoignaient en $\mathrm{Y}$ dans le massif du rempart. Il semble que le sol de ces cases ait été exhaussé sans qu'ait été comblée la conduite. En effet, le niveau de la dernière époque de la case I. 3 est marqué par l'area d'un pressoir, posé sur la canalisation après avoir été inversé. La même disposition a été retrouvée dans l'îlot V B I (cases 1 et 3), dont la canalisation, d'abord parallèle au mur de la case 3 , traverse en oblique la case 1 (fig. 18), pour déboucher au ras de la tour 5 par un orifice de 0,30 sur $0,60 \mathrm{~m}$, de dimension analogue à celui qui a été mis au jour à l'angle de la tour $6(0,40$ sur $0,50 \mathrm{~m})$.

Il semble que la ville haute ait été dotée du même système d'égouts sur le front est; il n'en subsiste qu'un orifice $(0,25$ sur $0,40 \mathrm{~m})$, débouchant au-dessus de l'assise de base ${ }^{27}$ à hauteur de la rue XIX ; l'arasement de la plus grande partie de ce rempart au-dessus des fondations en a fait disparaître tout vestige.

\section{Mobilier, círamique et technigues artisanales}

Le dégagement de la ville haute n'est pas encore assez étendu pour qu'il soit possible d'affirmer la différenciation sociale des deux quartiers : acropole, résidence des "princes" salyens, avec magasins et casernements (îlots XIII, XV et XX) et ville artisanale à fonction agricole et industrielle, ainsi que le fait apparaître la fouille du quartier situé entre les deux enceintes. C'est dans ce dernier secteur qu'ont été trouvés des pressoirs à huile, des outils agricoles et des fours, qui attestent l'activité agricole et industrielle de la population.

\section{a) Artisanal agricole.}

La technique oléicole est représentée par des dalles d'areae de pressoir, dont plusieurs sont en place (II. 1 ; I. 3; X. 4) et des contrepoids à rainure, tous incomplets, dispersés dans les rues (rues IX et XI ; cases XII. 4 ; XI. 10 ; X. 9), parfois remployés comme assise d'un poteau de bois destiné à soutenir la toiture (I. 7 ; X. 9 ; XI. 8) ; un de ces fragments remployés obturait l'entrée d'une case (XI. 9).

La mauvaise qualité de la roche locale (oligocène) avait rendu nécessaire la recherche de pierres de grande taille des carrières aixoises de Bibemus et de poudingue de Beauregard,

(27) Gallia, XXII, 1964, p. 574, fig. 3. 
pour la confection de plusieurs areae et de presque tous les contrepoids. L'utilisation de la première de ces carrières pour les piliers du sanctuaire remployés à l'époque d'Entremont II et III et le remploi de plusieurs éléments de pressoir font supposer que la technique de cette industrie était déjà connue de la première période. Acquise des Grecs de Varseille, elle n'est pas différente de ce qu'elle sera en Provence à l'époque romaine : les olives, entassée: dans des cabas ou des scouffins de sparterie empilés sur la dalle de l'area, étaient comprimées sous l'action d'un levier de bois, dont l'extrémité mobile était abaissée à l'aide d'un treuil maintenu au sol par un lourd contrepoids de pierre, dans les rainures duquel s'encastraient ses montants. Mais celui-ci agissait surtout par son poids; l'exiguïté de l'huilerie, en effet, ne permettait pas le logement d'un levier suffisamment long, dont la pression est d'autant plus forte et le maniement plus aisé qu'est éloigné son point d'appui ménagé dans le mur.

L'huile extraite du pressoir s'écoulait dans la gouttière circulaire de l'area et était dirigée par le déversoir dans des vases de terre cuite (X. 4). Cette technique, qui a été signalée à la même époque dans l'oppidum de la Courtine à Ollioules, ne connaît pas l'épuration dans des bassins bétonnés, qui sera courante à l'époque romaine ${ }^{23}$.

La mouture de la farine était faite à la main. Deux types de meules montrent son évolution : à la meule grecque rectangulaire et à trémie, enchâssée dans un bàti de bois, qui permettait de l'actionner par un mouvement de va-et-vient ${ }^{29}$, type rare à Entremont (niveau inférieur de la case XII. 4, fragments remployés dans les murs de la case X. 12) mais reconnu dans les habitats en relation avec les Grecs (Saint-Blaise, Arles, Mouriès, Les Pennes, Ollioules, Ensérune, Montlaurès, elc.), avait succédé la meule circulaire, à mouvement rotatif, courante dès la Tène II, provenant des carrières de lave de Beaulieu au nord d'Aix ou d'Ollioules.

Le tissage et le travail du cuir, dont l'importance est relevée par la minutieuse reproduction, dans la statuaire, des casques, des cuirasses, des pourpoints, des sandales, sont représentés par leur outillage : fusaïoles de terre cuite de type bitronconique, parfois ornées de dents de loup, pesons de tisserand de type pyramidal, aiguilles d'os, racloirs de fer.

\section{b) Artisanal industriel.}

L'artisanat industricl était développé, ainsi que l'indiquent les crassiers de minerai de fer, dispersés dans les rues (îlots VIII et I; rues I et IX; cases V B I. 2, etc.) et une grande plaque de plomb (ép. 0, 015-020 m) d'une centaine de kilos, découpée irrégulièrement sur les bords par des dentelures et des enroulements (0,73 sur $0,68 \mathrm{~m}$ ); elle a été trouvée dans le four circulaire (XI. 5), adossé à un four de plus grande dimension (XI. 6 et 7$)^{30}$, dont ne subsistent que les trois entrées en plein cintre, respectivement, du nord au sud, de $0,35,0,51$ et $0,47 \mathrm{~m}$ de hauteur sur $0,36,0,34$ et $0,50 \mathrm{~m}$ de largeur.

28) Ca. F. Brwort, Pressoirs d'olives à levier el à contrepoids en Provence el Afrique, dans Mémoires Institut histor. de Provence, 13, 1935, p. 106 ; J. LaYet, La Courline d'Ollioules La défense de la Courtine), 1949, p. 100.

(29) F. Brwort, Hisloire de l'outillage rural et arlisanal, 1947, fig. V, 3.

(30) Gallia, XVIII, 1960, p. 294, fig. 13 et XX, 1962, p. 691, fig. 8-10. 

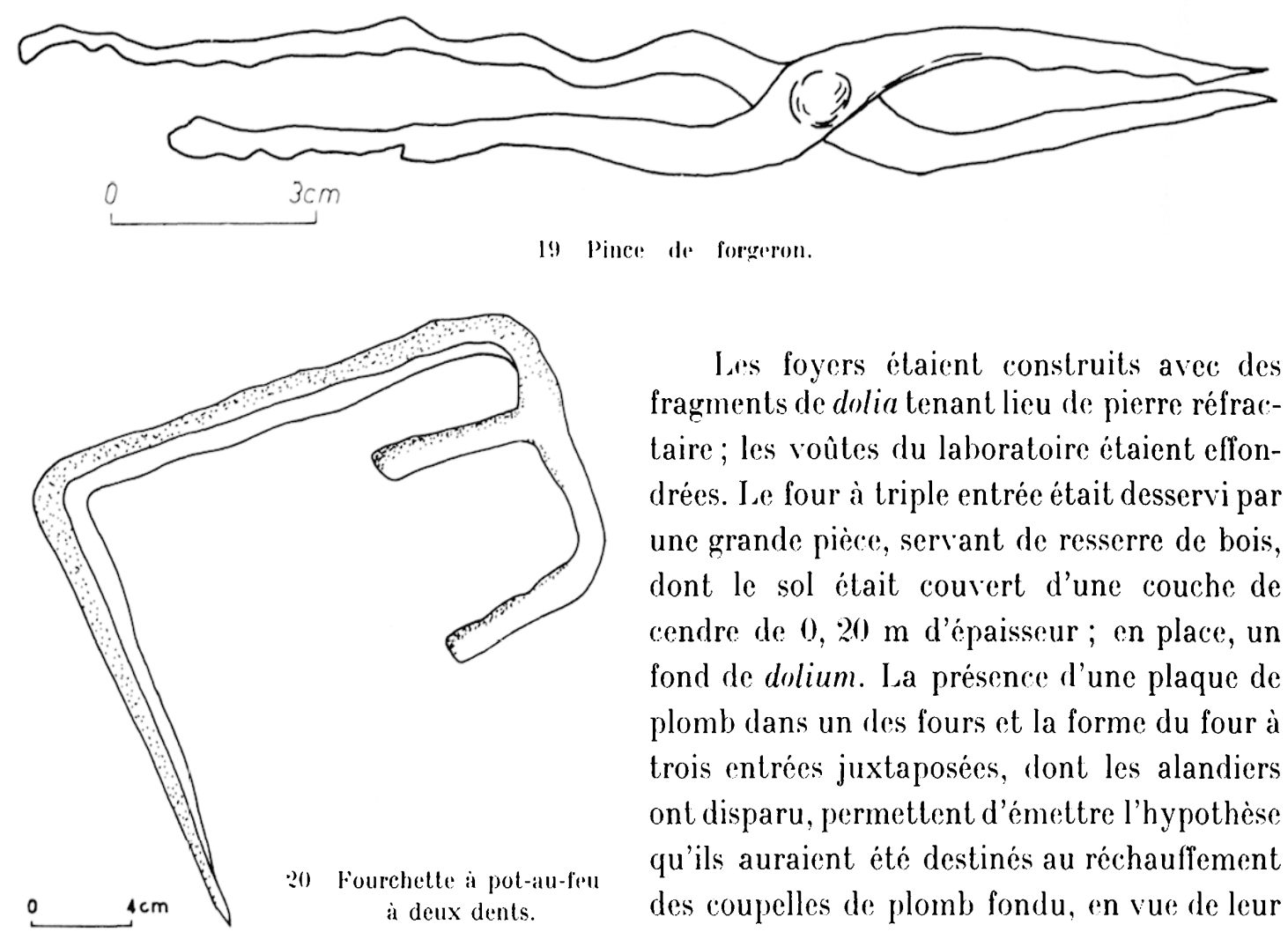

Les foyers étaient construits avec des fragments de dolia tenant lieu de pierre réfractaire; les voûtes du laboratoire étaient effondrées. Le four à triple entrée était desservi par une grande pièce, servant de resserre de bois, dont le sol était couvert d'une couche de cendre de $0,20 \mathrm{~m}$ d'épaisseur; en place, un fond de dolium. La présence d'une plaque de plomb dans un des fours et la forme du four à trois entrées juxtaposées, dont les alandiers ont disparu, permettent d'émettre l'hypothèse qu'ils auraient été destinés au réchauffement des coupelles de plomb fondu, en vue de leur utilisation. Son usage était en effet courant pour la réparation de la vaisselle et des dolia, à l'aide d'agrafes retrouvées en grand nombre, et pour la fabrication des balles de fronde.

Les importations de lingots de fer et de plomb provenant des mines de la montagne (Maures et Alpes), mais peut-être aussi des échanges avec Marseille, avaient donné naissance à une petite industrie locale, à laquelle on doit attribuer l'outillage artisanal (fig. 19 et 20 ) : faucilles, houe bidens, soc d'araire (I. 5), pour les travaux agricoles ; outils à douille (gouge, ciseau à bois, grattoirs), clous à tête aplatie, pattée, convexe, de toutes dimensions, pour les travaux de menuiserie; crochets (XI. 8 et 9); fourchettes à pot-au-feu coudées, à long manche $^{31}$ à deux ou trois dents (T. XIII et XVI ; rue I) ; couteau à lame droite (rue IX ; XI. 10) ou courbe (VIII. I) ; grattoir de 0, $07 \mathrm{~m}$ de long (VIII. 4); chaîne à maillons (XI. 9); piquet de fer avec anneau pour attacher un chien. en place, vers le milieu de la pièce (X. 11), elc.; des armes de guerre : partie supérieure d'un poignard de 0, 25 m de long (rue IX); pointes de javelots et lances (rue IX et enclave ouest).

c) Bronze el objels de parure.

Les objets en bronze, certains d'une excellente facture, étaient sans doute importés. A côté de boucles de ceinture sans décoration (XI. 2, 3), de nombreux anneaux (XI. 3 et 10 ; VIII. I et 4 ; X. 8 ; rue IX), de charnières de coffret percées de trous destinés à recevoir des clous (XI. 10), quelques pièces ornementées : une phalère en lunule décorée, en guise

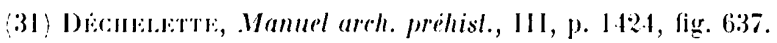



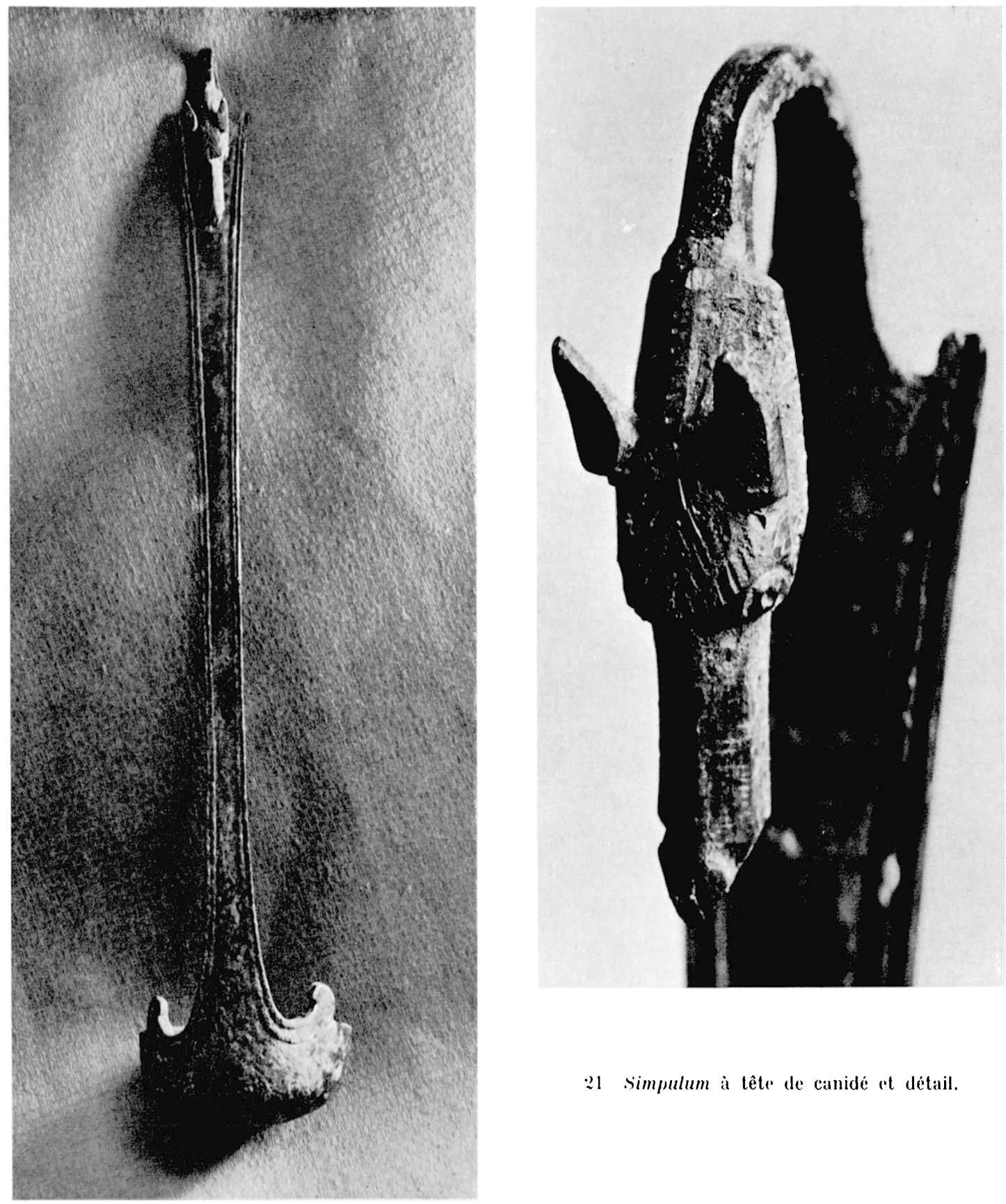

:1 Simpulum à tête de canidé et détail.

de gorgoneion, d'une "tête coupée" humaine ${ }^{32}$, analogue aux pièces de harnachement (phalères) de Manerbio (province de Brescia) et de Horovič en Bohême, du iII siècle et d'Augst, d'époque romaine ${ }^{33}$; une applique de ceinture décorée de rouelles, de type hallstattien (rue XVII) ${ }^{34}$; plusieurs fragments de simpulum à extrémité recourbée et un

(32) Gallia, V1II, 1950, p. 119, fig. 5.

33) W. Deovis, Phalères celliques el galio-romaines décorées de têles humaines, dans Rev. archéolog., 1950, I, pp. 35 ot 147 .

(34) F. Bkolt, Enlremonl, 1957, p. 20 (fig.). 
simpulum complet (long. $0,25 \mathrm{~m}$ ) orné de deux volutes, dont le manche est terminé par une tête de canidé (VIII. 2) (fig. 21), analogue aux nombreux exemplaires, généralement de moins bon style, du ${ }_{I_{1}} \mathrm{e} / \mathrm{I}^{\mathrm{er}}$ siècle, trouvés dans les nécropoles de Glanum, du Mont Menu à Eyguières (manche terminé par une tête de cygne), de Servane à Mouriès ${ }^{35}$; un passeguide en forme d'oméga terminé par deux têtes de canidés (X. 8) (fig. 22) $)^{35 \text { bis }}$; un fragment de tôle de bronze (cnémide ?) (X.12) et des fibules en bronze, à ressort (rue IX), appartenant à deux types différents : fibule en arbalète à ressort bilatéral (8 spires), à corde intérieure, de la Tène $\mathrm{I}^{36}$; fibule à arc filiforme à corde intérieure, à 4 spires, de la Tène II/III (fig. 23), dont le type susbistera à l'époque gallo-romaine ${ }^{37}$.

Les objets de parure sont rares et également importés : l'un des plus intéressants est une boucle d'oreille en fils d'argent torsardés et soudés, en forme de croissant fermé (case V B I. 1) (fig. 24), analogue à celui qui orne une tête de femme de la statuaire ${ }^{38}$. Ce type, de technique orientale, remontant au Ile millénaire en Phénicie, est connu en Occident par le pendant d'oreille en or du comptoir de Lattes, à l'embouchure du Lez, et par ceux de Banasa, d'importation phénico-punique ${ }^{39}$.

Le corail, provenant de Marseille, était travaillé sur place, ainsi que l'attestent des fragments bruts (rue IX; T. XIV; sondages dans la ville basse : T. XVI). Les perles de verre bleu, des fragments de bracelets de verre polychrome incrustés d'un filet en zigzag, sont une production courante des ateliers italo-celtiques. Cependant, doivent être attribuées à un travail local deux amulettes phalliques en os, avec trou de suspension, trouvées dans les cases X. 9 et I. 7 (fig. 25).

\section{d) Vaisselle.}

La principale industrie était celle de la poteric. Toutes les cases ont un ou plusieurs dolia, alignés le long des parois, parfois en place (VIII. 3 ; X. 5; X. 9 ; I. 2), la base légèrement enfoncée en terre. Ils sont renforcés au pied par un bourrelet, comme le pilhos grec ; en argile farcie de quartz, ils sont de fabrication locale. Leur panse peignée porte parfois, incisé avant cuisson, un signe apotropaïque : croix ; signe en S (VIII. 5), symbole de T'aranis, en omega ou en lyre (XI. 10); pentacle ${ }^{40}$, ces derniers également signalés sur les dolia de l'oppidum de Baou-Roux.

La vaisselle courante (fig. 26) était faite sur place : jattes à égouttoir, petits vases à libation en terre rose ou jaunâtre, gobelets peignés de tradition hallstattienne en terre noire, faits au tour, très abondants, parfois décorés d'une frise en "dents de loup » ou d'une $n^{\circ} 1748$.

(35) P. DE Bri:x, Mém. Instilut historique de Provence, 1931 ; Provincia, 1933; Rhodania, Congrès d'Arles, 1936,

(35) (fis. W. Kвӓмl: Lalènezeitliche Trensenanhänger in Omega/orm, dans Germania, 42, 1964, p. 25:2.

(36) I) ÉcheletTre, Manuel arch. préhisl., II, p. 853, fig. 352.

37) (f. sur ce tỵpe Dícheletre, o. c., III, p. 1253, fig. 535 ; L. L.trat, Les fibules gallo-romaines (Cat. des collections arch. de Besançon), 1956, pl. I, 18-20; R. GAvit.t., Fibules gallo-romaines recueillies à Lugdunum Convenarum, dans Ogam, XIV, 1962, p. 202, fig. 1 at 2.

(38) F. BlixolT, L'Art primilif médilerranéen, pl. 44, 1.

39) A. Jodix, Bijoux et amulettes du Maroc punique, dans Bull. Arch. marocaine, 6, 1966, p. 72 et pl. 6.

10 Ce signe, qui se trouve également à Entremont sur une coupe campanienne, est courant depuis le va siecle : amphore de Chios à Daphnae (J. BoARdmax, The Greelss Overseas, 1964, p. 147, fig. 38), coupe ionienne de .larseille (Ogam, 9, 1957, p. 108, fig. 2; Gallia, XXII, 1964, p. 576, fig. 7); vases attiqurs, amphore massaliete du Pigue, etc. 


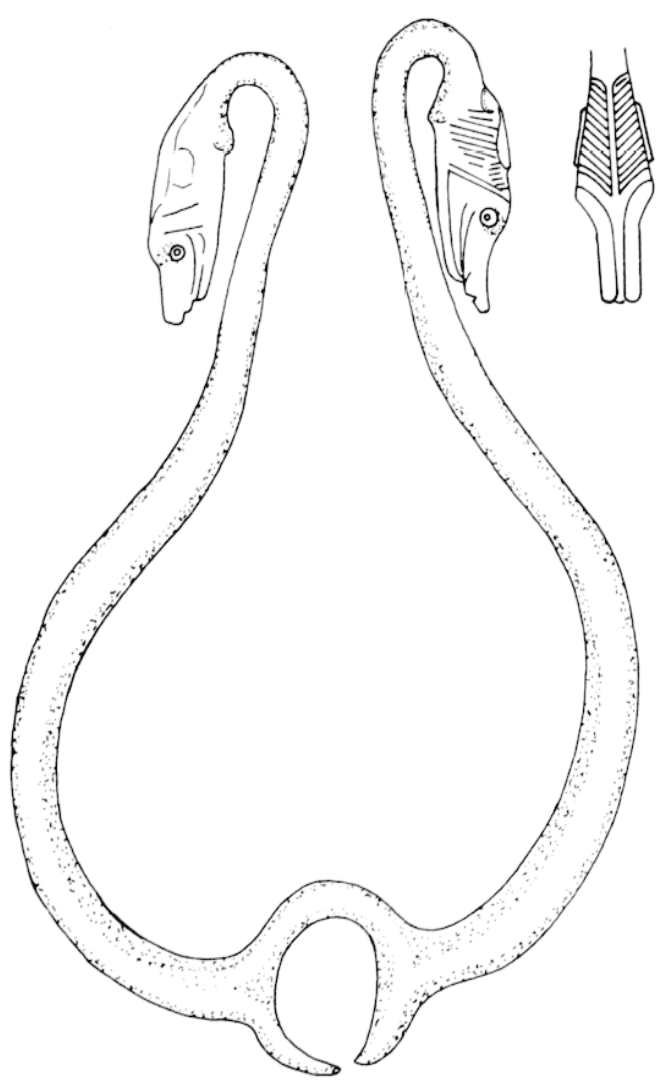

:2 Passe-guide en oméga à têtes de canidé, analogue à celui de Némi haut. 0, 095).
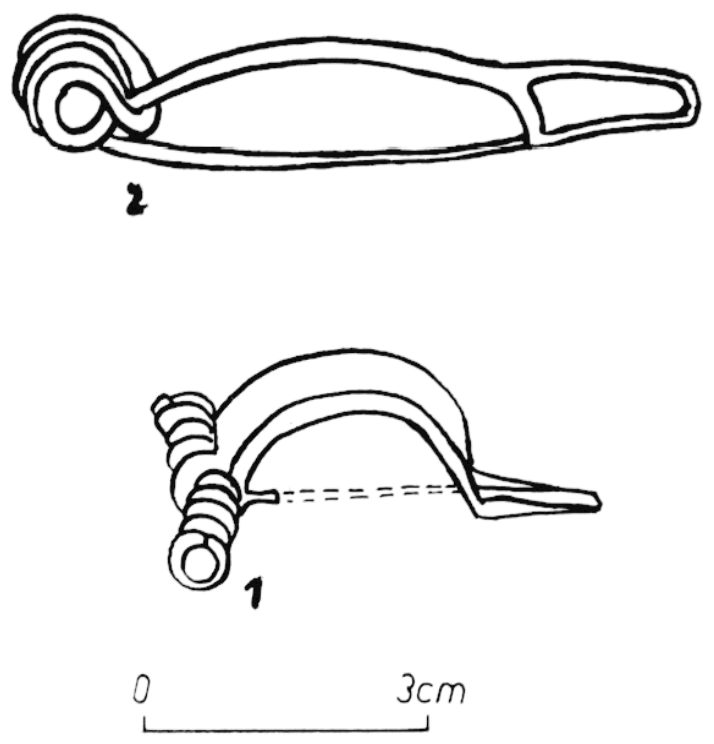

23 Fibules en bronze : 1 en arbalete; 2 a arc filiforme à corde intérieure.
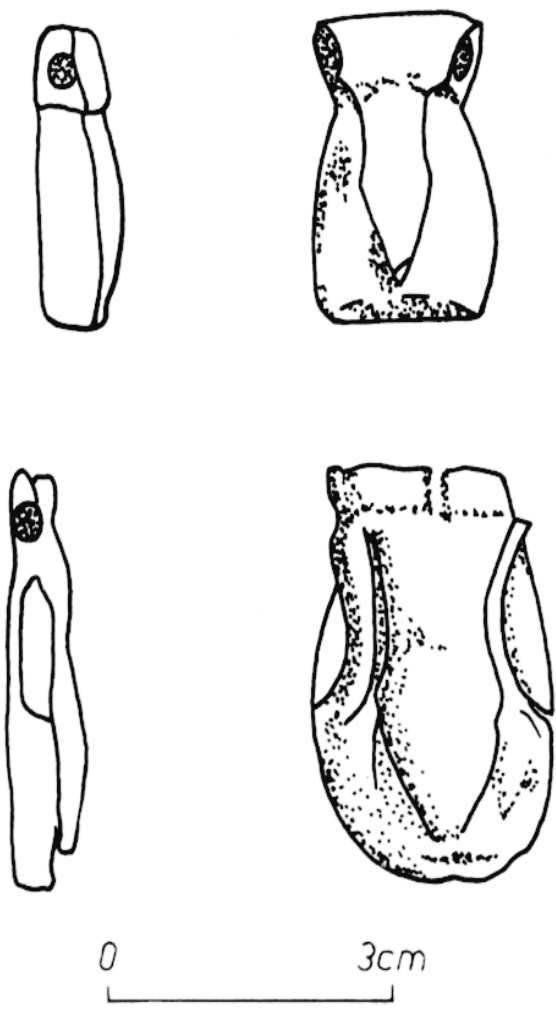

25 Amulettes phalliques en os.

24 Pendant d'oreille en fils d'argent torsadés diam. 0, 04). 

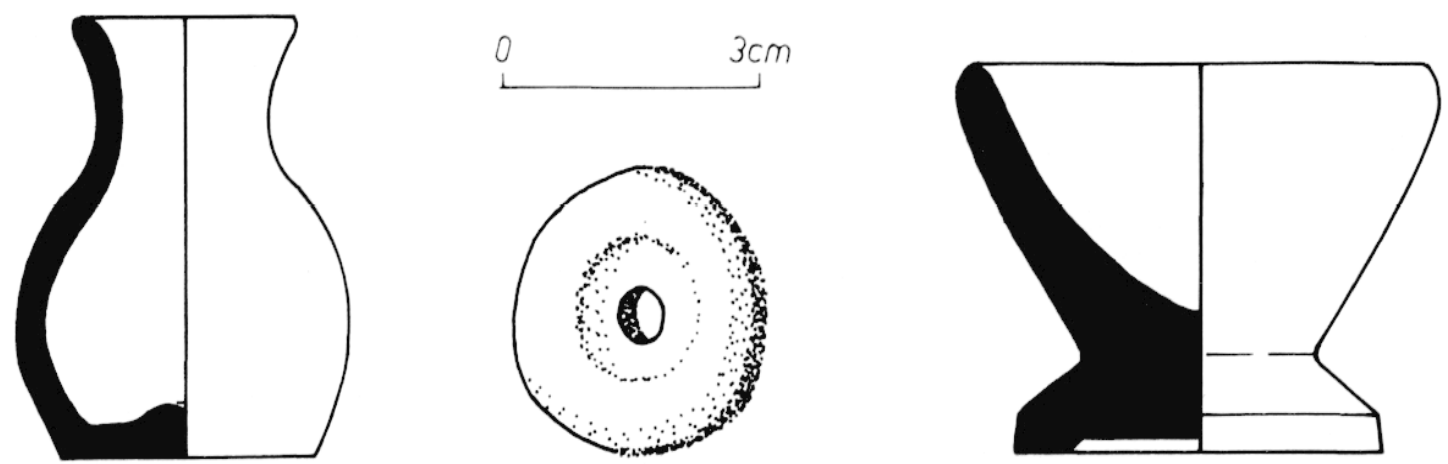

26 Vase à libation. Fusaiole. Petit vase indigène.

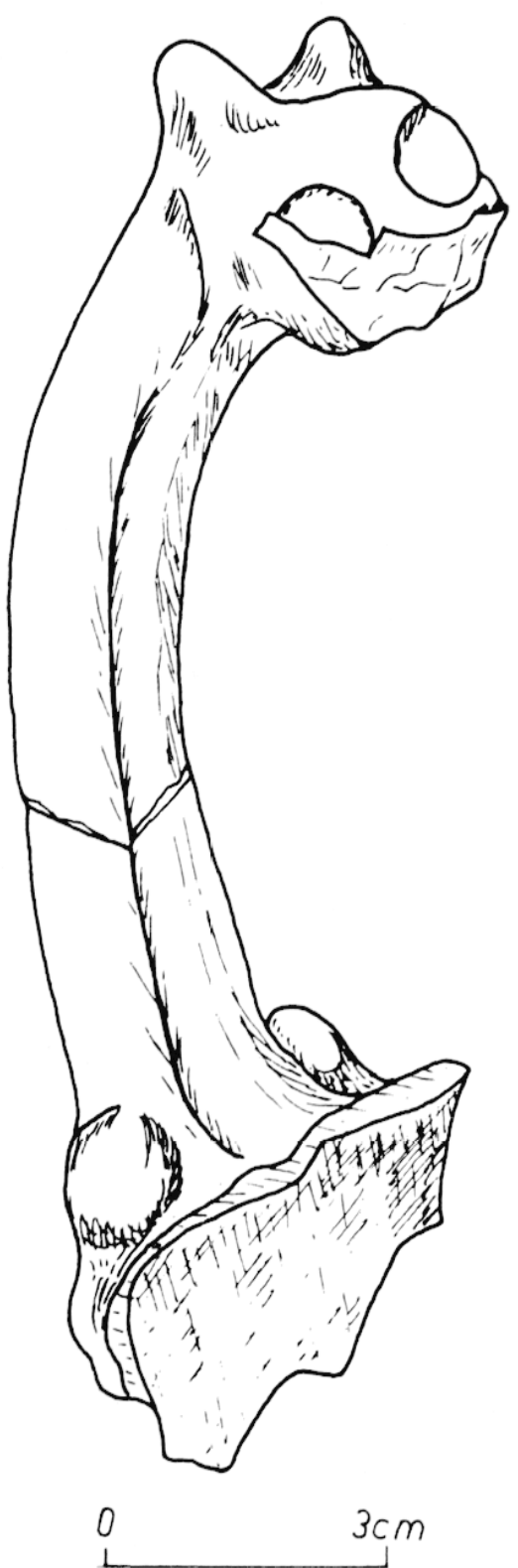

\&. 27 Anse de cruche zoomorphe.

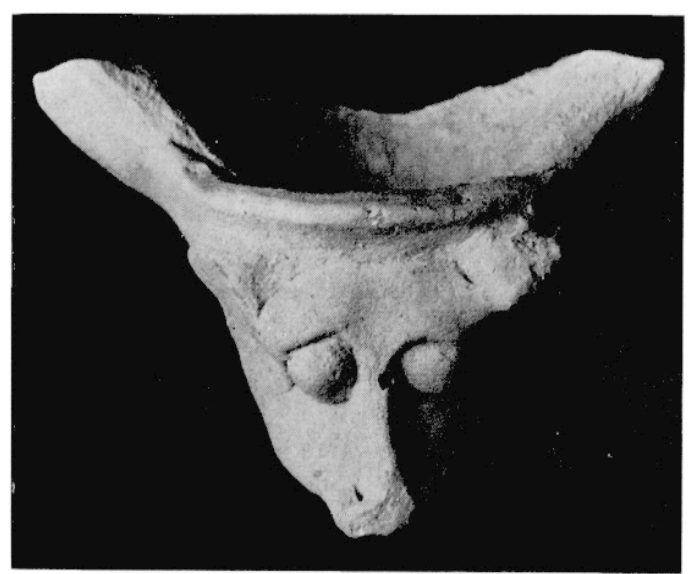

28 Bec verseur de pelvis à mufle de lion.

double ou triple rangée d' "ondes". Quelques pièces à décoration artistique zoomorphe se distinguent de cette production courante : des cruches à anse en pâte noirâtre ou grisâtre, finement lustrée, révèlent une tradition hallstattienne $e^{41}$, sans doute par imitation italo-étrusque. L'anse façonnée en corps étiré de canidé prend son attache, marquée par deux prolubérances, sur la panse du vase et se termine par une tête aux oreilles dressées qui se penche sur l'orifice du vase (voie sacrée) (fig. 27). Le bec verseur d'une pelvis en terre jaunâtre est façonné en mufle de lion (rue XI) (fig. 28), prototype des vases de Lezoux.

(41) Vase de Hongrie: P. Jacobsthal, Early Cellic Arl, 1941, p. 205 , fig. 418 . 


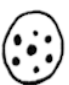

T. XIII 80

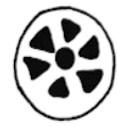

T. XIII (pre)

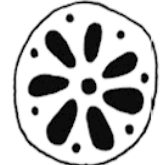

$\mathrm{XVI}(\mathrm{C})$

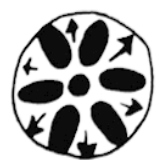

S.

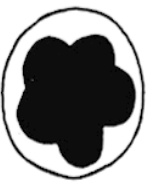

$X V I I 2$

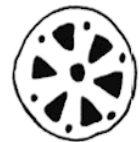

SANCT.

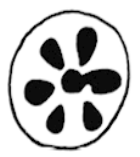

T. d.R
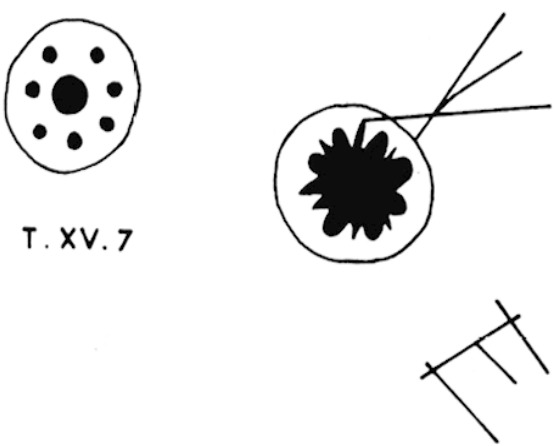

XIV 1

29) I)ecor de palmettes et de rosettes sur poterie campaniemne.

La rusticité de cette vaisselle contraste avec la finesse de la céramique campanienne à pâte rose (A) importée de Marseille : assiettes, coupes à décoration de palmettes et de rosettes (fig. 29), bols ornés d'une bande blanche, lampes à réservoir caréné (fig. 30), balsamaires à long col, représentent l'importation courante du ${ }_{\mathrm{II}} \mathrm{e}$ siècle, dont l'épave du Grand Congloué à Marseille nous a donné l'échantillonnage. On notera en particulier la coupe à bord rentrant et l'assiette à rebord horizontal peu incliné (fig. 31), qui appartient à la typologie du ${ }_{\mathrm{III}} \mathrm{e} / \mathrm{II}^{\mathrm{e}} \operatorname{siècle}^{42}$.

Cette vaisselle était appréciée : plusieurs pièces ont été restaurées avec des agrafes en plomb. La rareté des graffites : initiales en lettres grecques ( $\Pi, \Delta)$, croix de St-André, croix inscrite dans un cercle, traits divergents (fig. 32), elc., contraste avec la richesse épigraphique de la vaisselle campanienne des comptoirs grees de la côte, Marseille, Antibes (Vaugrenier), Olbia; à signaler, cependant, un graffite représentant un cheval tournant la tête en arrière (rue XVIII) (fig. 33), un nom incomplet en caractères grecs : $\Phi \mathrm{I} \Lambda \mathrm{H}\left(\mu \omega \nu\right.$ ? ${ }^{43}$ et un autre graffite portant à l'extéricur de la coupe les noms d'indigènes, le Grec Mardios, en cursive latine avec l'emploi de lettres grecques $(\mathrm{P}, \Lambda, \Upsilon)$ (fig. 34 et 35) : BAL (bus?). MARDILS. HR (mes?). CV....BAL.(bus?) (VBI. 2).

La poterie campanienne est contemporaine des bols hellénistiques à reliefs, provenant sans doute de la côte d'Asie Mineure, trouvés dans un sondage de prospection de la ville basse en $1956^{44}$ et d'un type de présigillée à bord rentrant, à vernis rouge, venant sans doute

(42) Formes Lamboglia $21 / 25$ (iallia, XVJ, 1958, p. 414) ot Lamboglia 6 = Grand Congloué 9 (iallia, XVIII, 1960, p. 292).

(43) Gallia, VIII, 1950, p. 119.

(44) Ibid., V, 1947, p. 82, fig. 2. Cf. F. BENort, L'épaue du Grand Congloué, p. 100, fig. 68 et A. I.ATMONivR, Bols grecs à reliefs à Toulouse, dans Pallas Annales de la Fac. des lettres de Toulouse), 3, 1967, p. 25. 

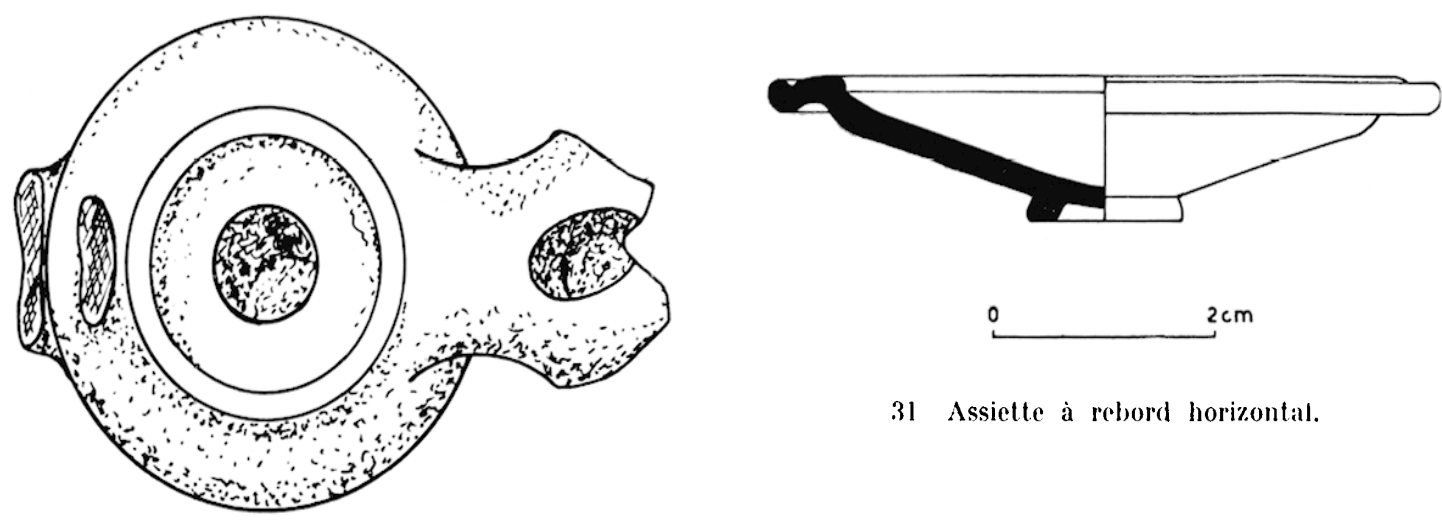
$2 \mathrm{~cm}$

31 Assiette à rebord horizontal.
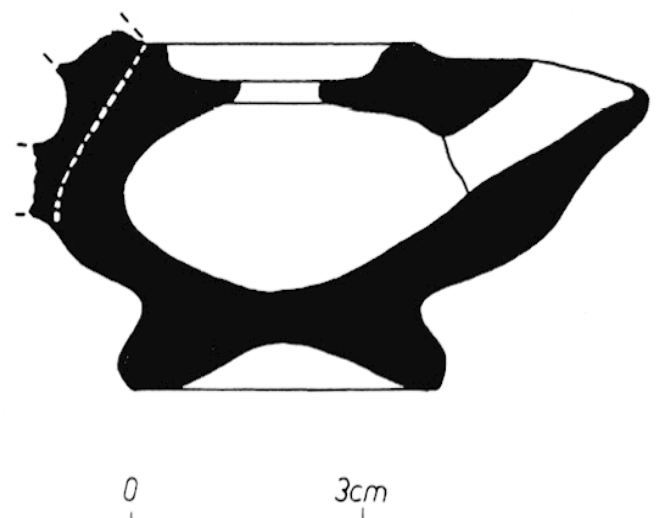

30 lampe campanienne à réservoir caréné.
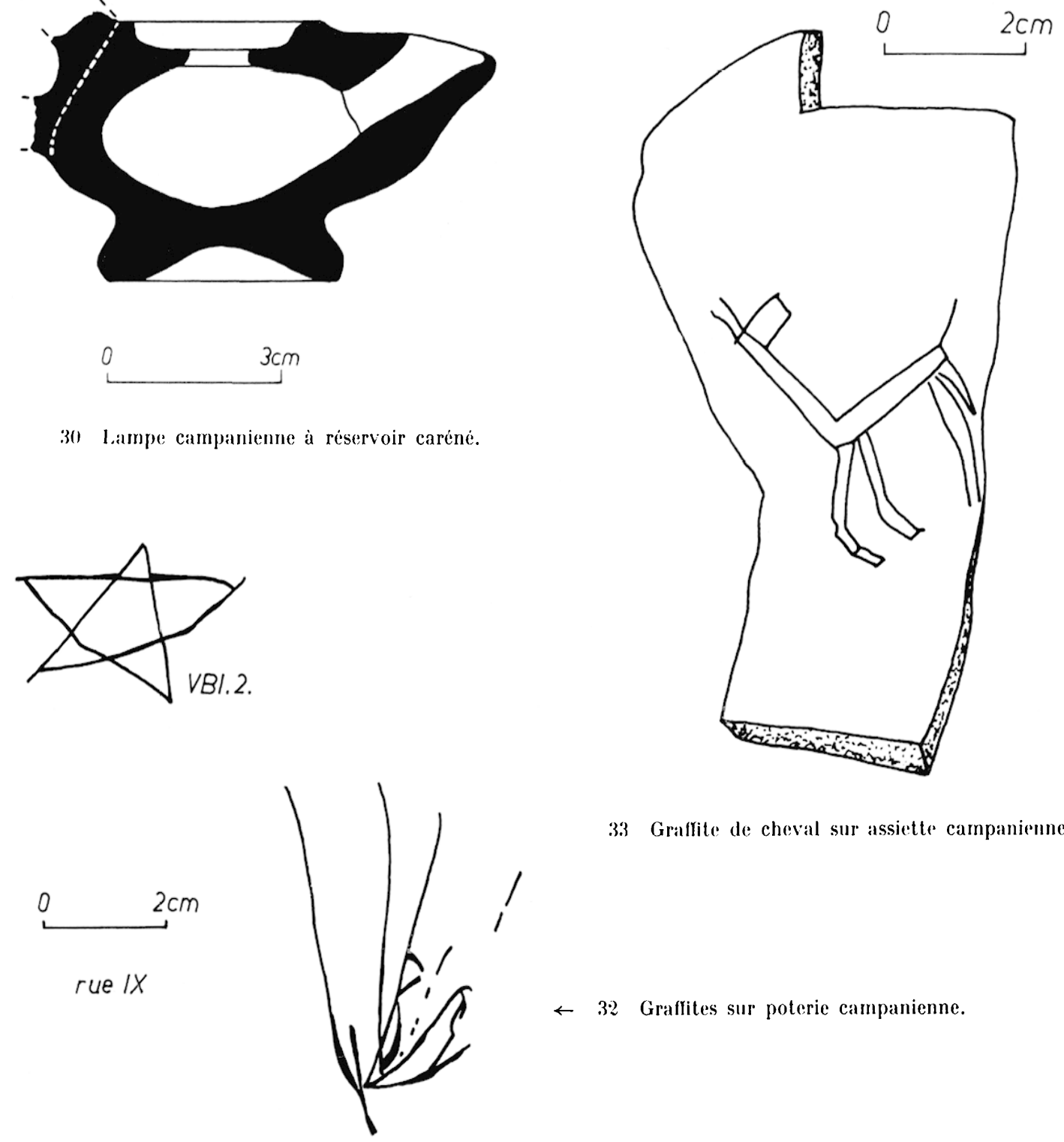

33 Graffite de cheval sur assiette campanienne.

$\leftarrow \quad 32$ Graffites sur poterie campanienne. 

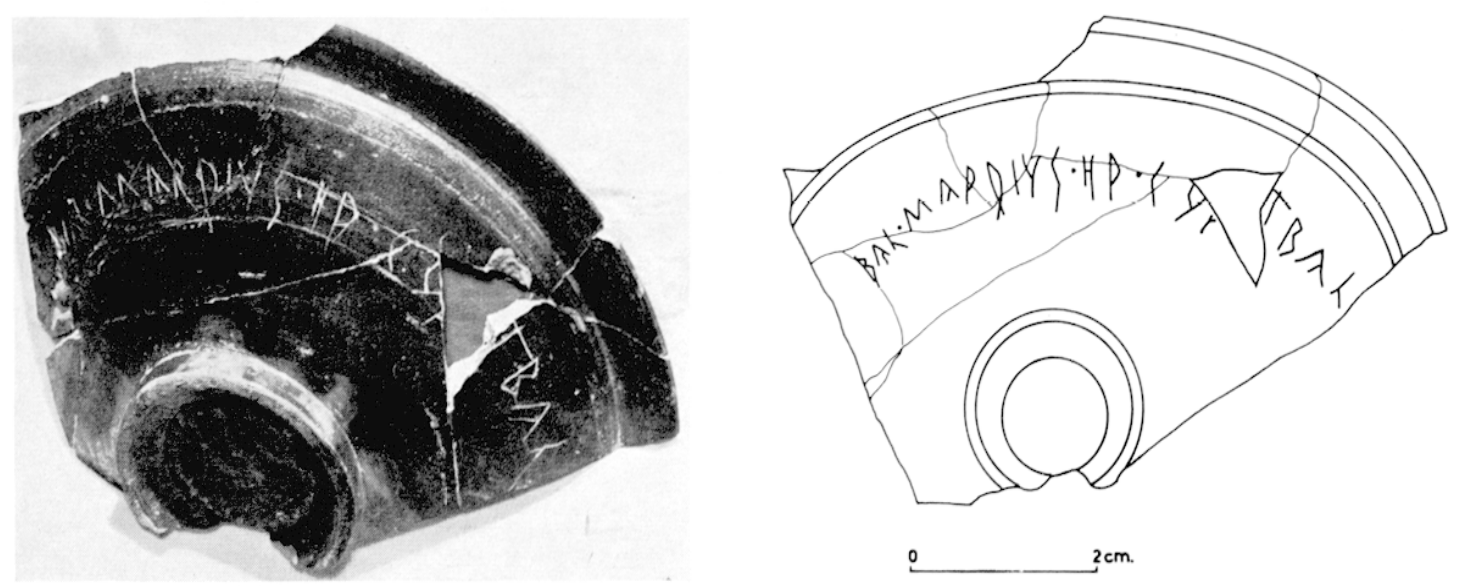

34 et 35 Coupe avec graffite anx noms de BAL. MARDILS. HIR. C: .. T BAI..

de Pergame, dont les tessons ont été recueillis, l'un dans les déblais de l'enceinte (tour 4), les autres, moins fins, dans la courche antérieure à l'incendie des cases I. 1 et 2 . Le matériel céramique de ces cases s'avère très riche : vases en terre non peignés, ornés de dents de loup, du type courant dans les deux quartiers ; céramique campanienne : vaisselle à pâte blanche marseillaise, dont un tesson, jusqu'ici unique, est décoré de languettes décolorées.

Il faut également signaler un type de poterie d'importation italique, le prototype du gobelet d'Aco, contemporaine de la campanienne A, ainsi que l'a révélé la fouille $d u$ Grand Congloué : le gobelet en poterie fine, à pied annulaire, à "décor clouté " (voie sacrée, rues IX et XI ; cases VIII. 2 ; XI. 3 ; XI. 9 ; XI. 10, elc.). Les plus anciens exemplaires, du ${ }_{\text {III }}{ }^{\mathrm{e}}$ siècle, ont été trouvés dans les nécropoles de Tuscania et de Castiglioncello au sud de Livourne, sur la côte tyrrhénienne; cette céramique est abondante dans les gisements ibériques antérieurs à 124 (Son Favar à Pollentia de Majorque, Ibiza, Ampurias) et a été signalée dans les oppida des Caisses à Mouriès et de Constantine (Lançon), ainsi qu'à Antibes, dans le dépotoir de l'anse Saint-Roch et au Grau d'Orgon en ('amargue ${ }^{45}$. Le type le plus ancien représenté à Entremont est caractérisé par une décoration linéaire formant une sorte de résille ou de filet pointillé, disposé en losanges aplatis alignés dans le sens horizontal. Son évolution, qui peut être suivie à Castiglioncello, tend vers un décor curviligne à arcatures et à volutes, dont un exemplaire a été trouvé à Entremont (rue IX).

De la côte catalane proviennent de petites urnes en terre grise, à haut col annelé, dont les fabriques avaient perpétuć la technique de la poterie grise monochrome phocéenne (îlots I et III du front ouest, rues IX et XI ; cases XI. 10, VIII. 2). Elles sont également associées à la poterie campanienne dans l'épave du Grand Conglouét6.

C'est également de la côte ibérique (Tarragone ?) qu'étaient exportés des bocaux cylindriques (sombrero de copa), sans doute de saumure, décorés de dessins linéaires rouges : dents de loup allongées à extrémité infléchie sur le marli; demi-cercles concentriques,

(45) Gallia, XIY, 1956, p. 221, fig. 61 ; L'epate de Grand Congloué, p. 103, fig. 69.

(46) Gallia, VIII, 1950, p. 119 ; XVII, 1960, p. 292. Cf. L'épave du Grand Congloué, p. 104, fig. 70 et pl. 15. 
dessins floraux sur la panse des sombrer $0^{47}$. Cette céramique est abondante sur le littoral languedocien du golfe du Lion et plus rare en Provence, où elle a ćté signalée à Marseille, à Saint-Blaise, à Glanum et dans les oppida de la région (Mouriès, Baou-Roux, le Castelet), à Antibes et à Nice (oppidum du Château). La stratigraphie de N. Lamboglia permet de lui assigner une date antéricure à 120 (rue XVII ; I. 3 ; VIII. 5 ; XII. 6). Des dessins floraux inédits la décorent.

Il faut enfin signaler, dans la ville haute et la ville basse ${ }^{48}$, quelques coupes en pâte claire rehaussées de bandes rouges ayant parfois viré au noir, dont le vernis, peu adhérent, est caractéristique de la production courante de Marseille à l'époque hellénistique (notamment à Roquepertuse).

e) Amphores.

Les importations les plus importantes étaient représentées par les amphores vinaires diffusées par les négociants marseillais; elles proviennent de Sicile et de Campanie (fig. 36) ; exceptionnellement, de Grèce.

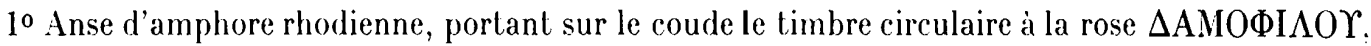
trouvée dans la couche supérieure de la ville basse (îlot VIII), de la première moitié du ${ }_{I^{e}}{ }^{\mathrm{e}}$ siècle ${ }^{49}$;

$2^{0}$ Amphore gréco-italique, de provenance syracusaine, caractérisée par son épaulement. curviligne, sa panse en forme de toupie, son pied terminé par un bouton et sa petite lèvre inclinée. Inconnue sur le limes rhénan, elle a été identifiée dans de nombreuses épaves sous-marines, en particulier celle du Grand Congloué (type républicain I).

I)eux timbres rétroverses TIBE (ri) sur l'anse (XI, 10 et rue XI) : la marque est connue à Trapani et au Mont Eryx ${ }^{50}$ et le nom abrégé TI. est associé à celui de Q. IV(v)ENTIVS sur une série d'amphores de l'épave du Grand Congloués1.

$3^{0}$ Amphore sud-italique, de Campanie, antérieure au type Dressel I courant sur le limes à l'épocque d'Auguste (type Dressel II ou républicain III); elle se distingue de celui-ci par sa lèvre courte, inclinée ou concave, son ogive fuselée, sans le pied massif de l'amphore également campanienne de Sestius ${ }^{52}$ :

C.L. FE(lix?), cachet rectangulaire sur le $\operatorname{col}^{53}$ (partie orientale XII. 1). Le cognomen FE est connu au Mont Beuvray ${ }^{54}$;

PI. M.R., également sur le col $(X .8)^{55}$;

$\mathrm{RV}(f i \text { ? })^{56}$, cachet rectangulaire sur l'anse (poterne de la ville basse);

I) IO(nis?), cachet rectangulaire sur l'épaule ${ }^{57}$;

47) .. Lambogtia, La ceramica iberica negli strali di Albintimilium e nel terrilorio ligure e tirrenico, dans Rev. ét. ligures, $\mathrm{XX}, 1954$, p. 88 , fig. 2, 1 (marli) ; fig. 4, 11-14. Cf. F. BForr, Recherches sur l'hellenisalion..., p. 85.

18) Rue XVII et îlot XVI (Gallia, VIII, 1950, p. 119), îlot I, cases 1 et 2.

49) Gallia, VIII, 1950, p. 119 ; L'épave du Grand Congloué, p. 3I, nº 2.

50) M. H. Callexder, Roman Amphorae, 1965, n 1721.

(51) L'épave du Grand Congloué, p. 38 ; Typologie el épigraphie amphoriques, dans Rev. ét. ligures, 23, 1957, p. 255.

(52) Typologie, l.c., p. 263 ; F. ZFvi, Appunli sulle anfore romane, dans Archenlogia classica, XVIII, 1966, p. 213.

53) Gallia, XII, 1954, p. 291.

54) Catlfexder, o. c., no 621 ; cf. Felix, no 623 (Rome, Ciarthage, Nîmes, Vienne, Xanten, elc.).

(55) PI signalé à Chambles (Loire), ibid., n $n^{\circ} 1328$.

(56) Ibid., $\mathrm{n}^{\text {os }} 1546-1548$.

(57) Ibid. nos 542-545. 

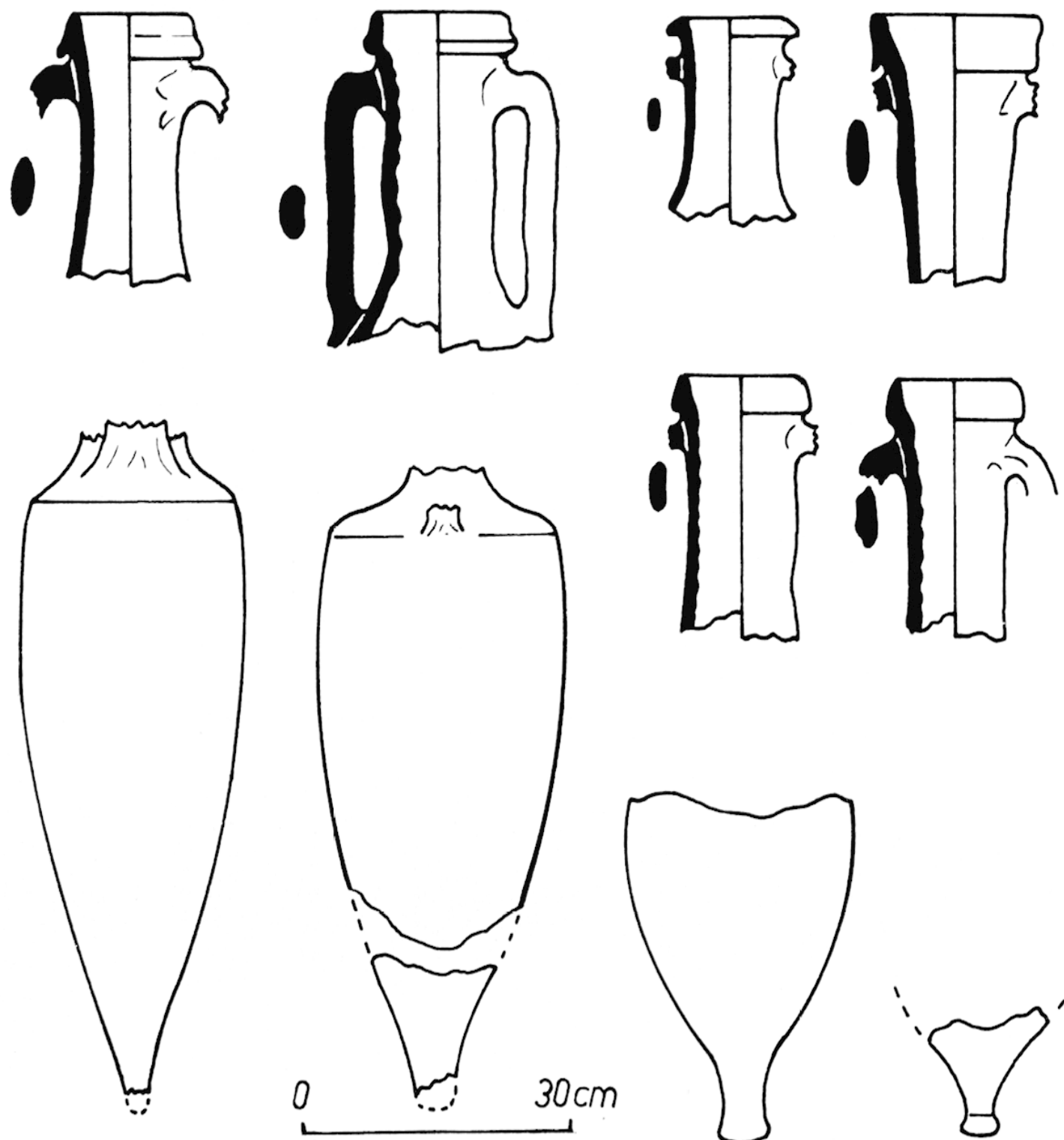

36
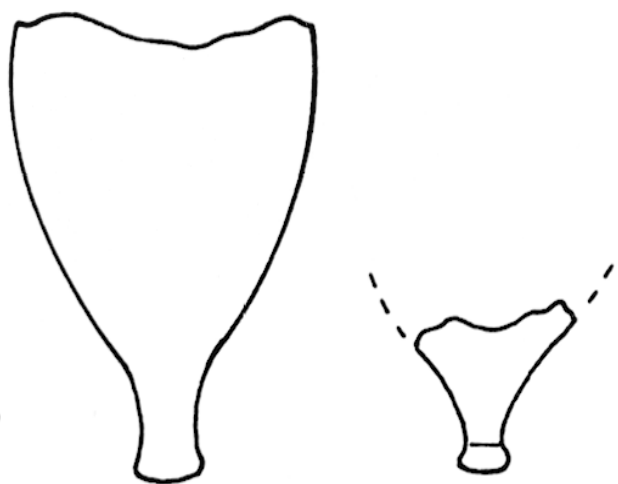

Amphores italiques et fonds d'amphores gréco-italiques.

I, cachet au haut de la panse (tranchée de sondage de la ville basse);

$\mathrm{s}$ (id.) ;

HIA, cachet rectangulaire sur la panse (voie sacrée);

$\mathrm{T}$, cachet rond au bas de la panse $\mathrm{e}^{58}$.

En outre, des chiffres peints en rouge, en partie effacés, se lisent sur le col de plusieurs amphores vinaires : VII, ..XII, LV, G XI. Étant donné leur variation, ces chiffres ne peuvent indiquer la contenance du liquide; leur numérotation indiquerait-elle le décompte du chargement au port d'embarquement (Pouzzoles ?) pour le paiement du droit de portorium ?59.

(58) Callender, o. c., no 1687 (Turin et le Puig près de Sagonte).

(59) Cf. F. Bexort, Signature de polier sur une coupe campanienne du Grand Congloué, dans Rev. èt. ligures, 28, 1962, p. 266. 
40 Deux cols évasés soulignés par un bourrelet, ayant appartenu à des amphores à saumure de Bétique, d'un type punico-romain ${ }^{60}$ caractérisé par l'adjonction d'un col et d'un pied, signalé a Alicante, Cadix, Lixus, Ruscino, elc. Elles proviennent d'une case aménagée dans la tour d'Entremont II (XII. 6) et de l'îlot III, limitrophe du front ouest.

La datation de ces amphores antérieurement à 125 et la présence de timbres ne comportant qu'un nom, parfois suivi de celui d'un associé, ont un grand intérêt pour la chronologie et la typologie de l'amphore qui a précédé le type augustéen (Dressel I); ces documents datés apportent un élément nouveau relatif à la complexité de la commercialisation du vin, dont l'exportation était parfois faite par plusieurs associés ${ }^{61}$.

\section{Échanges, monnaies}

Cies importations permettent d'esquisser la physionomie politique et sociale de l'arrière-pays massaliète. Elles témoignent des relations commerciales avec Marseille, qui en échange recevait du blé, des ovins, des peaux de bête, sans doute aussi des minerais rares (fer, plomb et argent), des herbes médicinales venant des Alpes et, vraisemblablement, des esclaves. Les trésors monétaires trouvés dans les cachettes des cases et les monnaies dispersées dans les rues à la suite du pillage, sont presque exclusivement constitués par le numéraire massaliète, drachmes et surtout oboles d'argent. L'une des cachettes, dans la ville haute, renfermait 1435 oboles; une autre, cent oboles, deux drachmes associées à un quinaire romain à l'effigie de la déesse Rome et au revers des Dioscures, et à deux victoriats à l'effigie de Jupiter et au revers de la Victoire (240-197) (case XX. 3) ; les deux autres trésors ont été trouvés dans le quarticr artisanal, l'un de 27 drachmes contenues dans une cassette en bois, (X. 9), l'autre, de 110 oboles que renfermait un petil pol de lerre (X. 8).

Des monnaies éparses ont été recueillies dans les rues et les cases : drachmes et petits bronzes de Marseille au taureau; denier de la famille Servilia (rue XI) : au droit, tête de Pallas casquée à droite, au revers C. SERVEILI. M.F., avec les Dioscures à cheval allant en sens contraire, de la première moitié du $\mathrm{II}^{\mathrm{e}}$ siècle $(M \text {. Serveilius a été consul en } 202)^{62}$; denier de la famille Flavia: au droit: Rome casquée à droite, au revers : Victoire sur un bige avec la légende Roma (couche supérieure de l'îlot X. 9).

La quantité du numéraire massaliète est en contraste avec l'extrême rareté des monnaies des Voconces et des Allobroges, dont aucune n'a été trouvée dans les cachettes de trésor (deux deniers d'argent à tête laurée à droite et bouquetin à droite, du poids de $2,35 \mathrm{gr}$ et $2,53 \mathrm{gr}$ (rue XVII), du type du trésor de Tourdan) ${ }^{63}$ et avec l'absence de numéraire arverne.

(60) Gallia, XVIII, 1960, p. 292, fig. 9 ; F. BExorr, Recherches sur l'hellénisalion de la Gaule méridionale, pp. 79 et 83 et pl. 42,1 et 2 ; cf. pl. 16, 3 .

(61) Cf. F. Benoit, Typologie el épigraphie amphoriques, l. c., p. 283.

(62) G. G. Belioxi, Le monete romane dell'età repubblicana, 1960, pl. 25, nos 625-628.

(63) A. BIAxcher, Trailé des monnaies gauloises, p. 269, fig. 127. A noter également une pièce d'argent (1, $90 \mathrm{gr}$ ) avec tête casquée et hippocampe, attribuée aux Allobroges (A. B LANCHET, o. c., p. 269, fig. 128). 
Les deniers de la République antérieurs à la prise d'Entremont sont également très rares : Entremont est sous la dépendance économique de Marseille. La composition des trésors montre l'importance des exportations en pays massaliète, les échanges avec les Allobroges, qui entretenaient cependant des relations d'amitié avec les Salyens, se faisant sans doute par troc. Les rapports avec Marseille, qui ne dépassaient guère le plan commercial, ne paraissent pas avoir exercé d'influence sur la vie spirituelle des Salyens, demeurés à l'état primitif avant la romanisation. On ne peut qu'être frappé par la quasi-absence de graffites grecs sur les céramiques ; l'inscription la plus importante sur une patère campanienne est en écriture cursive latine, la plus ancienne inscription de la Narbonnaise ${ }^{64}$.

Fernand Benoit.

(64) La plus ancienne inscription lapidaire latine étant le milliaire de Domitius : P.. M. Devar, A propos du milliaire de Cin. Domilius Ahenobarbus imperalor, dans Gallia, VII, 1949, p. 214. 